\title{
Emprego de Banco de Filtros e do Teorema de Imersão de Takens em Padrões Espaciais para a Classificação de Imagética Motora em Interfaces Cérebro-Computador
}

\section{Employing Filter Banks and Takens Immersion Theorem over Spatial Patterns to Motor Imagery Classification on Brain-Computer Interfaces}

\author{
Yule Vaz ${ }^{1,2}$ \\ Rodrigo Fernandes de Mello ${ }^{1,3}$
}

Data de submissão: 20/04/2016, Data de aceite: 09/10/2016

\begin{abstract}
Resumo: As Interfaces Cérebro-Computador (BCI) são sistemas que provêm uma alternativa para que pessoas com perda severa ou total do controle motor possam interagir com o ambiente externo. Para mapear intenções individuais em operações de máquina, os sistemas de BCI empregam um conjunto de etapas que envolvem a captura e pré-processamento dos sinais cerebrais, a extração e seleção de suas características mais relevantes e a classificação das intenções. Neste trabalho, diferentes abordagens para a extração de características de sinais cerebrais foram avaliadas, a mencionar: i) Padrões Espectro-Espaciais Comuns (CSSP); ii) Padrões Esparsos Espectro-Espaciais Comuns (CSSSP); iii) CSSP com banco de filtros (FBCSSP); e, finalmente, iv) CSSSP com banco de filtros (FBCSSSP). Em comum, essas técnicas utilizam de filtragem de banda de frequências e reconstrução de espaços para ressaltar similaridades entre sinais. A técnica de Seleção de Características baseada em Informação Mútua (MIFS) foi adotada para a redução de dimensionalidade das características extraídas e, em seguida, Máquinas de Vetores de Suporte (SVM) foram utilizadas para a classificação do espaço de exemplos. Os experimentos consideraram o conjunto de dados BCI Competition IV-2b, o qual conta com sinais produzidos pelos eletrodos nas posições $\mathrm{C} 3, \mathrm{Cz}$ e C4 a fim de identificar as intenções de movimentação das mãos direita e esquerda. Conclui-se, a partir dos índices kappa obtidos, que os extratores de características adotados podem apresentar resultados equiparáveis ao estado da arte.
\end{abstract}

Palavras-chave: Interfaces Cérebro-Computador, Imagética Motora, Padrões Espaciais, Teorema de Imersão de Takens, Eletroencefalografia

\footnotetext{
${ }^{1}$ Instituto de Ciências Matemáticas e de Computação (ICMC), Universidade de São Paulo (USP) - Av. Trabalhador Sãocarlense, 400, CEP 13560-970, São Carlos, SP, Brasil.

${ }^{2}$ \{yule.vaz@usp.br\}

${ }^{3}$ mellodicmc.usp.br\}
} 
Emprego de Banco de Filtros e do Teorema de Imersão de Takens em Padrões Espaciais para a Classificação de Imagética Motora em Interfaces Cérebro-Computador

\begin{abstract}
Brain-Computer Interfaces (BCI) are an alternative for people that suffer from severe or complete loss of motor control so they may have some interaction with the environment. To transform individual intentions into machine operations, BCIs rely on a series of steps that include brain signal acquisition and preprocessing, feature extraction, selection and classification. In this work, different approaches were evaluated for feature extraction: i) Common Spectral-Spatial Patterns (CSSP); ii) Common Sparse Spectral-Spatial Patterns (CSSSP); iii) Filter Bank Common SectralSpatial Patterns (FBCSSP); and, finally, iv) Filter Bank Sparse Spectral-Spatial Patterns (FBCSSSP). In common, those techniques use frequency-band filters and space reconstruction to highlight similarities. Mutual Information-based Feature Selection (MIFS) and Support Vector Machines (SVM) were also employed for the feature dimensionality reduction and classification, respectively. Experiments considered the dataset BCI Competition IV-2b, which provides signals collected using the electrodes $\mathrm{C} 3, \mathrm{Cz}$ and $\mathrm{C} 4$ in order to identify the movement intentions for the right and left hands. We conclude the feature extraction techniques considered in this work produce statistically equivalent results to the state-of-the-art.
\end{abstract}

Keywords: Brain-Computer Interfaces, Motor Imagery, Spatial Patterns, Takens Immersion Theorem, Electroencephalography

\title{
1 Introdução
}

O cotidiano de um indivíduo é permeado pela execução de diversas tarefas consideradas essenciais em sua vida, tais como sua comunicação e sua movimentação. Essas interações com o ambiente externo são determinadas por suas intenções e executadas a partir de suas atividades sensório-motoras, as quais exigem a comunicação entre Sistemas Nervoso Central, Periférico (SNC e SNP, respectivamente) e músculos. O bloqueio da transmissão de sinais entre o SNC e SNP ocasiona em total ou severa perda do controle motor de um indivíduo, tal como ocorre naqueles com Esclerose Lateral Amiotrófica (do inglês Amlyotrophic Lateral Sclerosis - ALS) [1].

Visando eliminar a necessidade de meios físicos para a interação entre um usuário e uma máquina, as Intefaces Cérebro-Computador (do inglês Brain-Computer Interfaces $\mathrm{BCI}$ ) surgem como alternativa para que esses indivíduos contem com um meio de interagir com o ambiente ao seu redor, melhorando, consequentemente, sua qualidade de vida. Neste contexto, as BCIs visam, a partir do imageamento da atividade cerebral do usuário, classificar suas intenções em operações posteriormente executadas por uma máquina criando, assim, um canal de comunicação alternativo ao natural.

As intenções de um indivíduo são tipicamente identificadas a partir de sinais cerebrais específicos denominados sinais de controle [2], que podem ser adquiridos por duas estra- 
Emprego de Banco de Filtros e do Teorema de Imersão de Takens em Padrões Espaciais para a Classificação de Imagética Motora em Interfaces Cérebro-Computador

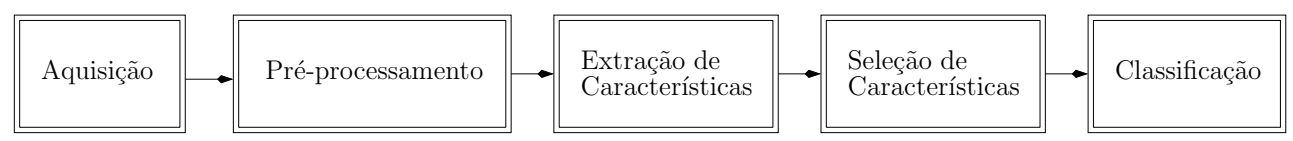

Figura 1. Ilustração das etapas envolvidas para a classificação de sinais de controle em Interfaces Cérebro-Computador.

tégias: Atenção Seletiva (AS) e Imagética Motora (IM). Os mecanismos que propagam as diferentes atividades cerebrais relacionadas à AS e à IM definem a complexidade e o modo com que cada operação de máquina será executada. Mais específicamente, a AS busca, a partir de sinais de controle exógenos (i.e., oriundos de estímulos externos), identificar itens sobre os quais um usuário mantém sua atenção, de forma que a própria BCI deve emitir os estímulos enquanto classifica a resposta produzida pelo cérebro, sendo necessária a sincronia entre essas duas tarefas [2, 3]. Cabe ainda ressaltar que, por ter uma natureza involuntária e pouco subjetiva, os sinais de controle adquiridos pela Atenção Seletiva são mais facilmente identificáveis se comparados aos adquiridos por IM. Além disso, as BCIs desenvolvidas por meio da AS abordam operações mais complexas tais como comunicação [3] e movimentos mais elaborados, contudo previamente definidos.

Em contrapartida, a estratégia de IM não necessita de sincronização já que adota sinais de controle endógenos (i.e., oriundos da própria intenção motora do indivíduo), os quais não requerem quaisquer estímulos externos para serem produzidos, garantindo maior liberdade para o usuário. Neste contexto, a Imagética Motora pode ser utilizada para o desenvolvimento de BCIs que funcionem em tempo real. Um sistema de BCI, tal como ilustrado na Figura 1. exige uma série de etapas: i) aquisição dos sinais cerebrais; ii) pré-processamento; iii) extração e seleção de características; e, finalmente, iv) classificação.

Na primeira etapa (aquisição), é necessário que as sessões experimentais sigam um formato bem definido de maneira que seja possível associar sinais cerebrais às classes de interesse. Emprega-se, tipicamente, o esquema de ensaios ilustrado pela Figura 2, os quais se iniciam com um período de referência em que o usuário é alertado sobre uma tarefa que deverá ser executada. Posteriormente, inicia-se o período de Imagética Motora no qual a tarefa determinada pelo sistema é efetuada (e.g., imagina-se o movimento da mão esquerda ou direita), sendo associadas aos sinais produzidos. Finalmente, um intervalo de tempo aleatório, que decorre até o próximo ensaio, é destinado ao relaxamento do usuário a fim de evitar sua adaptação ao sistema [4].

Para o monitoramento das atividades cerebrais durante essas sessões experimentais, técnicas de imageamento cerebral tais como Eletroencefalografia (EEG), Espectroscopia do Infravermelho Próximo (do inglês Near Infrared Spectroscopy), Magnetoencefalografia (MEG), 
Emprego de Banco de Filtros e do Teorema de Imersão de Takens em Padrões Espaciais para a Classificação de Imagética Motora em Interfaces Cérebro-Computador

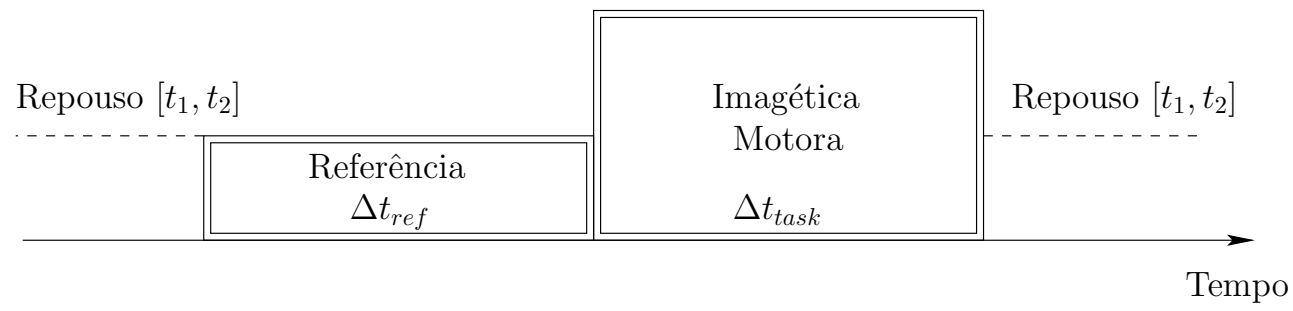

Figura 2. Os ensaios são iniciados com um período de espera, denominado repouso. Posteriormente, o indivíduo é avisado que haverá uma tarefa a seguir (período de referência). Logo após, a tarefa é apresentada e executada pelo indivíduo o qual, finalmente, é submetido a um período de repouso de tempo aleatório.

Ressonância Magnética Funcional (do inglês Functional Magnetic Resonance Imaging fMRI) e Eletrocorticografia (ECoG) podem ser empregadas para a coleta dos dados [2]. Como a EEG é a que apresenta maior mobilidade e menor custo financeiro, além de não exigir procedimentos clínicos invasivos, ela é amplamente empregada em trabalhos encontrados na literatura [2, 4, 5]. Essa técnica faz uso de eletrodos posicionado sobre o escalpo humano para monitorar a atividade neuroelétrica. Tipicamente, adota-se os padrões 10-20, 10-10 e 10-5 para se definir a disposição desses sensores, tal como ilustrado na Figura 3 Cada um desses capta a atividade neuroelétrica produzida em uma região com uma grande densidade de neurônios e, portanto, apresenta baixa resolução espacial.

Apesar do menor custo financeiro dos equipamentos de Eletroencefalografia, a coleta de dados ainda requer uma infra-estrutura laboratorial específica a qual nem todos têm acesso. Contudo, a competição BCI Competition criada a partir do projeto Berlin Brain-Computer Interface [4] conta com a publicação de dados (i.e., sinais cerebrais) de sessões experimentais efetuadas para a análise de diversos problemas envolvidos nas BCIs. Dentre tais problemas, este trabalho aborda o BCI Competition IV-2b, a fim de comparar diferentes abordagens de extração de características de sinais de controle por meio de seus índices kappa. Os sinais considerados foram captados a partir de três eletrodos de EEG, posicionados em C3, Cz e C4 (maiores detalhes na Figura 3), e três de Eletrooculografia (EOG). Esses sensores de EOG mapeiam a atividade ocular do indivíduo facilitando a remoção de eventuais artefatos.

Cabe ainda ressaltar que a técnica de EEG apresenta alta sensibilidade a ruídos tais como artefatos exógenos produzidos pela variação de impedância dos eletrodos [7], e endógenos, produzidos por outras atividades do indivíduo tal como movimentação ocular [8]. Esses artefatos devem ser eliminados a fim de melhorar a qualidade dos sinais de controle e, consequentemente, a acurácia da classificação. Tipicamente, emprega-se filtros estreitos 
Emprego de Banco de Filtros e do Teorema de Imersão de Takens em Padrões Espaciais para a Classificação de Imagética Motora em Interfaces Cérebro-Computador

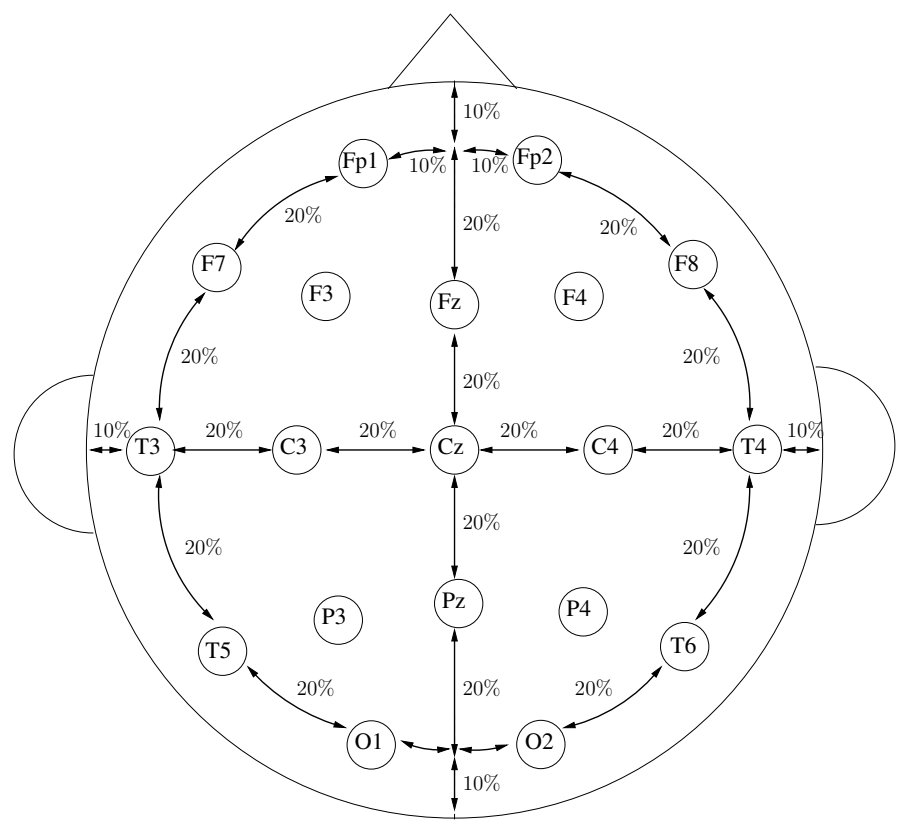

Figura 3. Posicionamento de eletrodos segundo o padrão 10-20 para o monitoramento eletroencefalográfico (Adaptada de [6]). Cada círculo rotulado corresponde a um eletrodo. Os rótulos indicam: a letra $\mathrm{F}$ corresponde à região frontal do escalpo humano; a letra $\mathrm{C}$ está associada à região cortical; a letra $\mathrm{T}$ indica a região temporal; a letra $\mathrm{P}$ corresponde à região parietal; e, finalmente, a letra $\mathrm{O}$ indica a região occipital.

(do inglês notch filter), de 50 ou $60 \mathrm{~Hz}$, na fase de pré-processamento, ou blinda-se o equipamento de EEG para a redução da influência da impedância dos eletrodos sobre os sinais adquiridos pela EEG [8]. Para complementar, artefatos endógenos, tais como os produzidos pelas atividades oculares (monitorados por Eletrooculografia - EOG), musculares (por Eletromiografia - EMG) e cardíaca (por Eletrocardiografia-ECG), podem ser eliminados na fase de aquisição pela prevenção de ensaios, instruindo o voluntário a evitar algumas atividades musculares e cardíacas. Contudo, certas ações são involuntárias e, portanto, inevitáveis. Além disso, inibir a atividade ocular tende a produzir variações indesejadas segundo [9] e [10].

Pode-se também, na fase de pré-processamento, remover todo o período em que os sinais sofreram interferência dos artefato, rejeitando, assim, todo um ensaio. Contudo, essa abordagem exige, tipicamente, que um especialista identifique os dados afetados. Ademais, mesmo com a automatização deste procedimento de detecção, há a perda de todo um ensaio 
Emprego de Banco de Filtros e do Teorema de Imersão de Takens em Padrões Espaciais para a Classificação de Imagética Motora em Interfaces Cérebro-Computador

que poderia conter informações sobre a Imagética Motora. Portanto, uma outra abordagem, conhecida como remoção de artefatos, efetuada na fase de pré-processamento, adota técnicas de filtragem ou decomposição, tais como Filtragem, Regressão e Combinação Linear, Análise de Componentes Principais (do inglês Principal Components Analysis - PCA) e Análise de Componentes Independentes (do inglês Independent Component Analysis - ICA), a fim de eliminar essas influências buscando evitar a perda de informações relevantes para a classificação de Imagética Motora [8]. Neste trabalho, emprega-se a ICA para a remoção de artefatos oculares dos sinais de EEG, visto seus bons resultados reportados pela literatura [11], e é considerado o trabalho de [11] para a identificação automática dos artefatos de EOG.

Posteriormente, a fase de extração de características é executada a fim de produzir informações relevantes sobre os sinais de controle, tais como os considerados pela Imagética Motora: a Dessincronização e a Sincronização Relacionada a Eventos (do inglês Eventrelated Desynchronization e Synchronization - ERD e ERS, respectivamente) das ondas $\mu$ e $\beta$ propagadas na região do córtex motor pela intenção de movimento, as quais estão presentes, respectivamente, nos intervalos de frequência $(7-12) \mathrm{Hz}$, e $(12-30) \mathrm{Hz}$ [3, 5]. Além disso, encontra-se na literatura, o uso do intervalo de $(4-40) \mathrm{Hz}$ para a caracterização dessas ondas [12], o qual é considerado neste trabalho.

Contudo, há sub-bandas dentro desses intervalos de frequência que apresentam maior relevância na classificação de Imagética Motora e, além disso, o comportamento espectral da ERD e ERS podem variar dentre diferentes indivíduos [13, 14, 15, 16] ao qual, portanto, o modelo de classificação de uma BCI deve se adaptar [5, 17]. Neste contexto, trabalhos como os de [18],[12] e [19] buscam encontrar configurações espectrais que melhor representem a Imagética Motora para uma pessoa específica. Cabe ainda ressaltar que as variações que caracterizam as ERDs são ainda mais evidentes em regiões contralaterais ao movimento. $\mathrm{O}$ oposto, ou seja, o aumento da atividade rítmica sensório-motora, ocorre após a movimentação ser executada ou imaginada, i.e., no período de relaxamento [5], e é chamada de Sincronização Relacionada a Eventos (ERS).

Tais características espaciais e espaço-temporais motivaram o desenvolvimento das técnicas de Padrões Espaciais Comuns (do inglês Common Spatial Patterns - CSP) [20], Padrões Espectro-espaciais Comuns (do inglês Common Spectral-Spatial Patterns - CSSP) [21] e Padrões Espectro-Espaciais Comuns Esparsos (do inglês Common Sparse Spectral-Spatial Patterns - CSSSP) [22]. O CSP visa encontrar transformações lineares que maximizem a variância dos sinais associados a uma classe de IM (e.g., movimentos da mão esquerda) e minimizem a dos associados a outra classe (e.g., movimentos da mão direita), produzindo um filtro que realce suas características espaciais. Ademais, o CSSP e o CSSSP, consideradas neste trabalho, visam aplicar o mesmo processo de otimização considerando a imersão dos sinais de controle, definida pelo Teorema de Imersão Takens [23], sobre um espaço de número de dimensões embutidas de, respectivamente, $m=2$ e $m \geq 3$, realçando seus atributos 
Emprego de Banco de Filtros e do Teorema de Imersão de Takens em Padrões Espaciais para a Classificação de Imagética Motora em Interfaces Cérebro-Computador

espaciais e temporais.

Há ainda outros trabalhos que reportam bons resultados na classificação de Imagética Motora para a base de dados BCI Competition IV-2b. Dentre eles, o trabalho [24] apresenta melhor índice kappa médio $(\kappa=0,66)$ empregando os Campos Aleatórios Condicionais Escondidos (do inglês Hidden Conditional Random Fields - HCRF) em conjunto ao CSP para extração de características, e modelos Autorregressivos (do inglês Autoregressive models AR) para tratamento de artefatos. Gaur et al. [25] adotam a Decomposição Empírica de Modos (do inglês Empirical Mode Decomposition - EMD) reconstruindo os sinais a partir das Funções de Modos Intrínsecas (do inglês Intrinsec Mode Functions - IMF) cuja frequência mais relevante se encontra no intervalo de $(6-24) \mathrm{Hz}$, i.e., de maneira que as IMFs representem as ondas $\mu$ e $\beta$. Utilizaram, posteriormente, as medidas de Hjorth [26] e as energias dos sinais contidos nos intervalos de frequência $(6-12) \mathrm{Hz}$ e $(16-24) \mathrm{Hz}$ para fornecer o espaço de características sobre o qual se empregou a Análise de Discriminante Linear (do inglês Linear Discriminant Analysis - LDA) para a classificação da IM.

A abordagem vencedora da BCI Competition IV-2b, [12], considera o uso de banco de filtros (do inglês Filter Banks - FB) antes da aplicação do CSP sobre os sinais de controle. Os autores filtram os sinais em nove diferentes sub-bandas de frequência de mesma largura, contidas no intervalo de $(4-40) \mathrm{Hz}$, e sobre cada qual, posteriormente, são encontrados os padrões espaciais pelo CSP. Além disso, segundo [12], o uso do CSSP e do CSSSP é desnecessário em conjunto com banco de filtros pois já aplicam um modelo autorregressivo sobre os sinais de controle. Contudo, da perspectiva do Teorema de Imersão de Takens [23], o CSSP e CSSSP em conjunto com FBs (FBCSSP e FBCSSSP) caracterizariam as imersões das sub-bandas, podendo levar a melhores resultados na classificação da Imagética Motora. Cabe ressaltar que Ang e Quek [12] ainda apontam que essas duas técnicas devem ser parametrizadas, logo, este trabalho considera a automatização deste processo.

As técnicas Common Spectral-Spatial Patterns (CSSP), Common Sparse SpectralSpatial Patterns (CSSSP), Filter Bank Common Sectral-Spatial Patterns (FBCSSP) e Filter Bank Sparse Spectral-Spatial Patterns (FBCSSSP) tendem a produzir um espaço de características com um grande número de atributos, o qual sofre, portanto, influência da maldição da dimensionalidade [27, 28]. Esse fenômeno acarreta em perda de acurácia (ou índices kappa) em termos de classificação, já que o aumento das dimensões de um espaço pode ocasionar a compactação dos dados [28]. Portanto, diversas técnicas tais como PCA, LDA e Seleção de Características baseada em Informação Mútua (do inglês Mutual Information-based Feature Selection - MIFS) podem ser empregadas para reduzir a quantidade de atributos do espaço de características, sendo a última adotada neste trabalho. Após este procedimento, utilizase abordagens de Aprendizado de Máquina, tais como Redes Neurais Artificiais (RNA), $k$ Próximos Vizinhos (do inglês $k$-Nearest Neighbors $-k$-NN) e Máquinas de Vetores de $\mathrm{Su}$ porte (do inglês Support Vector Machines - SVM), para, finalmente, classificar a Imagética 
Emprego de Banco de Filtros e do Teorema de Imersão de Takens em Padrões Espaciais para a Classificação de Imagética Motora em Interfaces Cérebro-Computador

Motora. No contexto deste trabalho, as SVMs são empregadas visto que, segundo o Limite de Máxima Margem [29], resultado produzido pela Teoria do Aprendizado Estatístico formulada por Vapnik com auxílio de Chervonenkis [30, 31], elas provêem garantias mais justas de aprendizado.

Este trabalho está organizado em: Seção 2 que apresenta detalhes sobre o conjunto de dados BCI Competition IV-2b; Seção 3 , que descreve a fase de pré-processamento dos sinais cerebrais; Seção 4 , a qual define as abordagens de extração e seleção de características empregadas; Seção 5 , que conta com a descrição dos experimentos e seus resultados produzidos e; finalmente, a Seção 6 discorre sobre as conclusões deste trabalho.

\section{BCI Competition IV-2b}

A BCI Competition IV-2b disponibiliza sessões de mapeamento cerebral efetuadas por EEG sobre nove voluntários destros e com visão saudável, abordando o problema de Imagética Motora referentes ao movimento das mãos esquerda e direita. Os eletrodos de EEG foram dispostos nas posições $\mathrm{C} 3, \mathrm{Cz}$ e C4 (para maiores detalhes veja a Figura 3 ) e adotouse também, a fim de facilitar o tratamento de artefatos de EOG, três eletrodos posicionados na face do indivíduo como ilustra a Figura 4, em que dois deles são colocados na lateral externa aos olhos e outro na região frontal, entre os olhos, captando sinais no intervalo de amplitude de $\pm 1 \mathrm{mV}$. Os sinais cerebrais e de atividades oculares foram adquiridos a uma taxa de frequência de $250 \mathrm{~Hz}$.

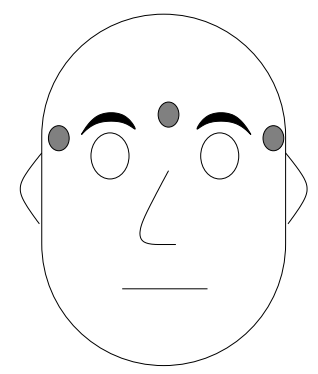

Figura 4. Disposição dos eletrodos que efetuaram a Eletrooculografia. Imagem adaptada a partir da descrição da base de dados $2 b$, encontrada na competição BCI Competition IV [4].

Efetuou-se, para cada indivíduo, cinco sessões experimentais para a aquisição dos dados. As três primeiras, $1 \mathrm{~T}, 2 \mathrm{~T}$ e $3 \mathrm{~T}$, destinadas ao treinamento do modelo de classificação de $\mathrm{IM}$ e as duas últimas, $4 \mathrm{E}$ e $5 \mathrm{E}$, ao seu teste. Cabe ressaltar, que dentre as três primeiras, a $1 \mathrm{~T}$ e a $2 \mathrm{~T}$ não contam com uma resposta ao usuário sobre o quão correto o sistema está interpretando suas intenções, i.e., não há feedback, e seus sinais foram adquiridos dentro do 
Emprego de Banco de Filtros e do Teorema de Imersão de Takens em Padrões Espaciais para a Classificação de Imagética Motora em Interfaces Cérebro-Computador

intervalo de amplitude de $\pm 100 \mu \mathrm{V}$, enquanto que na 3T foram adquiridos no intervalo \pm 50 $\mu \mathrm{V}$. Cada uma das sessões se iniciou pelo mapeamento da atividade ocular dos voluntários que eram instruídos a ficarem dois minutos com os olhos abertos, observando uma cruz de fixação na tela, um minuto com os olhos fechados e um minuto movimentando-os. A aquisição dos sinais de EOG do indivíduo foi dividida em quatro ensaios de 15 segundos, com cinco segundos de intervalo entre cada bloco.

Cada uma das sessões $1 \mathrm{~T}$ e $2 \mathrm{~T}$ contam com um total de 120 ensaios referentes à Imagética Motora da mão esquerda e da mão direita sobre a qual o indivíduo selecionou, antes do inicio do experimento, o que ele acreditava ser a forma mais natural de se executar. Cada ensaio iniciou com a apresentação da cruz de fixação na tela e um estímulo acústico de aviso, emitido na frequência de $1 \mathrm{kHz}$ durante $70 \mathrm{~ms}$. Alguns segundos depois, foi apresentada na tela, durante 1,25 segundo, a tarefa a ser executada, a qual, por meio de uma seta, indicava para qual mão deveria-se imaginar o movimento. Neste contexto, o voluntário contava com 4 segundos para completar a tarefa. Após o término do ensaio, houve um período de repouso médio de 1,5 segundo adicionado a um intervalo aleatório de até um segundo, a fim de evitar a adaptação do indivíduo.

As três sessões com feedback online consistiram de ensaios que apresentavam a intensidade com que o indivíduo imaginava o movimento determinado pelo sistema. Ao início de cada ensaio, um smiley cinza era apresentado no centro da tela para indicar nenhuma atividade. Após dois segundos do início do ensaio, um curto sinal auditivo era emitido. A tarefa era dada no intervalo de 3 até 7,5 segundos. O usuário era instruído a mover o smiley para a esquerda ou para a direita dependendo da Imagética Motora. Se a execução da IM estivesse correta, a cor da imagem tornava-se verde, caso contrário, vermelha. No instante de $7,5 \mathrm{se-}$ gundos, a tela do monitor tornava-se vazia e o voluntário esperava um período aleatório de um a dois segundos para o início de um novo ensaio. O esquema dos ensaios com e sem feedback são ilustrados na Figura 5 .

Cabe ressaltar que a BCI Competition IV-2b adota o índice kappa de Cohen [32] para avaliar e comparar o desempenho dos trabalhos participantes. Este índice apresenta o quão distante um classificador responde, em termos de acurácia, de uma decisão aleatória. Ademais, a BCI Competition emprega apenas filtros estreitos de $50 \mathrm{~Hz}$ e passa-bandas na faixa de frequência de $(0,5-100) \mathrm{Hz}$, não efetuando a remoção de artefatos de EOG a qual, neste trabalho, é abordada por meio da técnica de Análise de Componentes Independentes (ICA).

\section{Pré-processamento}

A ICA permite a decomposição de sinais multivariados em outros considerados como suas fontes, as quais são denominadas Componentes Independentes (do inglês Independent Components - ICs) e interpretadas como elementos estatisticamente independentes. Essa 
Emprego de Banco de Filtros e do Teorema de Imersão de Takens em Padrões Espaciais para a Classificação de Imagética Motora em Interfaces Cérebro-Computador

a)

Imagética Motora Imagética Motora

da mão esquerda da mão direita
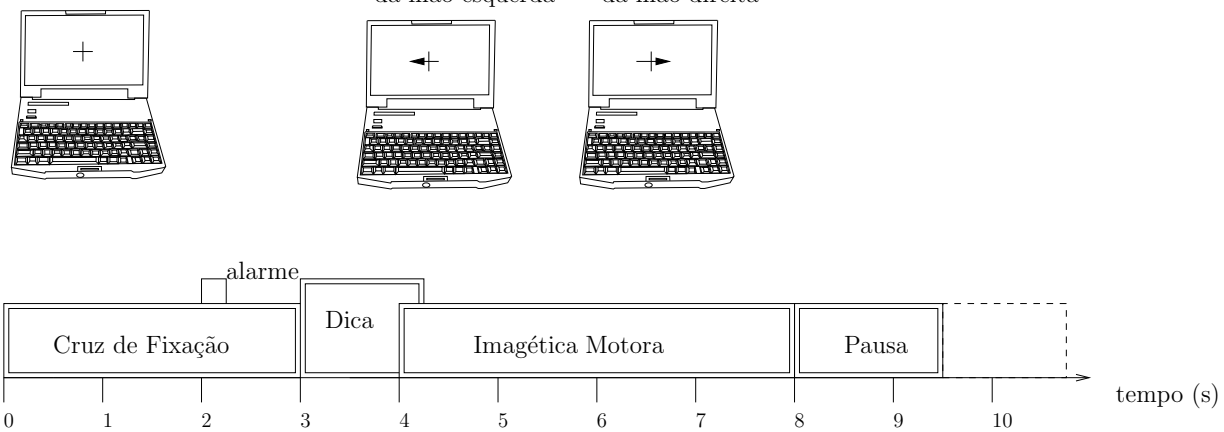

b)
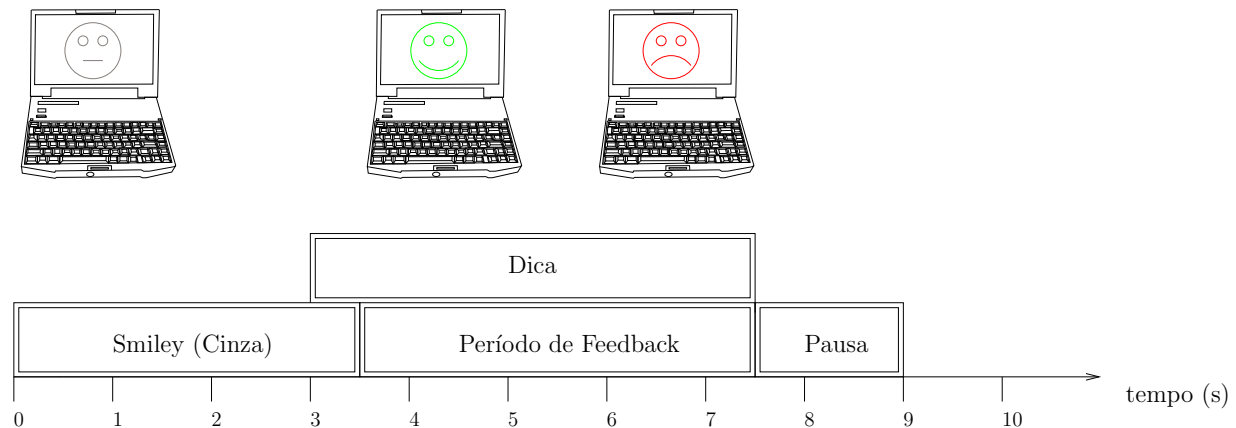

Figura 5. Esquema de ensaios utilizado pela $B C I$ Competition IV-2b: a) ilustra a estrutura de um ensaio efetuado nas sessões sem feedback (1T e 2T); e b) nas sessões com feedback (3T, 4E e 5E). Ilustração adaptada de [4].

técnica produz solução para o problema Cocktail Party em que uma conversa entre duas pessoas é capturada por dois sensores e deseja-se, a partir das gravações, extrair a fala de cada uma delas. Para isso, a ICA busca encontrar uma transformação linear $\mathbf{P}=\mathbf{A}^{-1}$ que produza os ICs, tal como apresentado pela Equação 1. em que A é a matriz de síntese dos sinais fontes, $\mathbf{S}$ os Componentes Independentes e $\mathbf{X}$ os dados originais compostos pelas 
Emprego de Banco de Filtros e do Teorema de Imersão de Takens em Padrões Espaciais para a Classificação de Imagética Motora em Interfaces Cérebro-Computador

aquisições $x_{c}(t)$ dos canais $c \in C$ de EEG e EOG tal que $x_{c}(t) \in \mathbf{X}$.

$$
\mathbf{X}=\mathbf{A S}
$$

Neste contexto, considera que os ICs são determinados ao assegurar que a transformação linear adquirida a partir de $\mathbf{P X}$ produzem variáveis com distribuições de probabilidades diferentes da distribuição Normal [33]. Para isso, utiliza-se, tipicamente, técnicas de gradiente para otimizar a Negentropia ou a Curtose dos sinais produzidos pela transformação P.

Em BCIs, é considerado que alguns desses ICs podem conter os elementos referentes aos artefatos de EOG, os quais são removidos no processo de síntese apresentado na Equação 1. Contudo, a ICA não conta com quaisquer maneiras de identificar quais Componentes Independentes contém esses artefatos, exigindo sua inspeção visual. É apresentado em [11] uma abordagem que automatiza essa identificação comparando os ICs aos sinais adquiridos pelos eletrodos de EOG por meio da correlação de Pearson, de forma que se o IC é desconsiderado na síntese dos novos dados $\tilde{x}_{c} \in \tilde{\mathbf{X}}$ se essa correlação for maior que um limiar $\theta$ para qualquer um dos sinais de EOG. Cabe ressaltar que, neste trabalho, a ICA é efetuada nos sinais extraídos de cinco segundos antes do início dos ensaios até o seu término.

\section{Extração e Seleção de Características}

Após a remoção de artefatos dos sinais de EEG, adota-se extratores de características para produzir informações relevantes para a classificação de Imagética Motora. Neste contexto, diversos trabalhos visam encontrar elementos espectrais, espaciais e temporais que melhor se adaptem a um indivíduo específico [12, 18, 19, 24, 25]. Neste trabalho, uma janela de tempo de tamanho $t_{f}$ de um ou dois segundos é extraída de $\tilde{\mathbf{X}}$ meio segundo após o início do período de Imagética Motora, resultando nos sinais $\mathbf{Z}$ (tal como ilustra a Figura 6). Sobre $z_{c} \in \mathbf{Z}$ são aplicados, primeiramente, filtros passa-bandas na faixa de frequências de $(4-40)$ $\mathrm{Hz}$ abrangendo as ondas $\mu$ e $\beta$. Posteriormente, emprega-se uma dentre as técnicas CSSP, CSSSP, FBCSSP e FBCSSSP para a extração de características dos sinais de controle.

O CSSP é uma extensão do CSP em que é considerada a imersão dos sinais de controle (i.e., séries temporais) sobre um espaço fase de número de dimensão embutida $m=2$ e coordenada de atraso arbitrária $\tau$ [23, [21]. A partir dos espaços de imersão $z_{c, \tau}(t)=\left(z_{c}(t), z_{c}(t-\tau)\right) \in \mathbf{Z}_{\tau}$ dos sinais $z_{c} \in \mathbf{Z}$, o CSSP calcula matrizes de covariância $\Sigma_{y_{i}}^{e}$ para cada ensaio $e \in E_{y_{i}}$ do conjunto de treinamento, associado a uma das duas classes $\left\{y_{1}=+, y_{2}=-\right\} \in \mathcal{Y}$, tal como apresentado na Equação 2 em que $N$ é o comprimento 
Emprego de Banco de Filtros e do Teorema de Imersão de Takens em Padrões Espaciais para a Classificação de Imagética Motora em Interfaces Cérebro-Computador

Imagética Motora

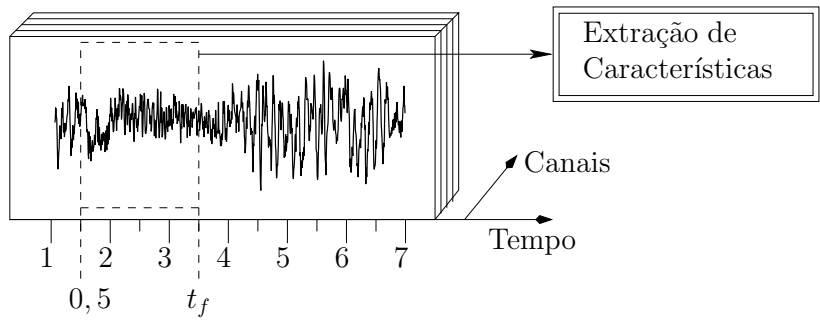

Figura 6. Janela de tempo utilizada na extração de características.

dos sinais.

$$
\boldsymbol{\Sigma}_{y_{i}}^{e}=\frac{1}{N}\left(\mathbf{Z}^{e}-\overline{\mathbf{Z}}^{e}\right)^{T}\left(\mathbf{Z}^{e}-\overline{\mathbf{Z}}^{e}\right) \text { para } e \in E_{y_{i}}
$$

Posteriormente, estima-se as matrizes de covariância média $\overline{\boldsymbol{\Sigma}}_{+}$e $\overline{\boldsymbol{\Sigma}}_{-}$associadas a cada classe tal como na Equação 3 .

$$
\overline{\boldsymbol{\Sigma}}_{y_{i}}=\frac{1}{\left|E_{y_{i}}\right|} \sum_{e \in E_{y_{i}}} \boldsymbol{\Sigma}_{y_{i}}^{e}
$$

Assim, calculam-se os filtros espaço-temporais $\left\{\mathbf{w}_{j} \in \mathbf{W}^{*} \forall j=c\right\}$ a partir do problema de autovetores generalizados, em que $\bar{\Sigma}_{+} \mathbf{w}_{j}=\lambda_{j} \bar{\Sigma}_{-} \mathbf{w}_{j}$, sendo $\lambda_{j}$ um autovalor e $\mathbf{w}_{j}$ o seu autovetor associado, solucionando a Equação 4.

$$
\mathbf{W}^{*}=\underset{\mathbf{W}}{\arg \max } \frac{\mathbf{W}^{T} \boldsymbol{\Sigma}_{+} \mathbf{W}}{\mathbf{W}^{T} \boldsymbol{\Sigma}_{-} \mathbf{W}}
$$

Cabe ressaltar que, pelo Quociente de Rayleigh, os filtros espaço-temporais encontrados a partir da Equação 4 maximizam $\boldsymbol{\Sigma}^{(+)}$enquanto minimizam $\boldsymbol{\Sigma}^{(-)}$. Espera-se então, que a transformação linear $\mathbf{W}^{*} \mathbf{Z}_{e}$, realce as características espaço-temporais das ERDs e ERSs para cada ensaio.

Contudo, o CSSP aborda apenas imersões com número de dimensões embutidas $m=$ 2 que podem não ser suficiente para a representação do espaço fase de uma série temporal. Isto motivou a proposta do CSSSP que considera um maior número de dimensões embutidas para o cálculo da Equação 4 , de forma que a imersão de $z_{c} \in \mathbf{Z}$ é determinada por 
Emprego de Banco de Filtros e do Teorema de Imersão de Takens em Padrões Espaciais para a Classificação de Imagética Motora em Interfaces Cérebro-Computador

$z_{c, m, \tau}(t)=\left(z_{c}(t), z_{c}(t-\tau), z_{c}(t-2 \tau), \ldots, z_{c}(t-(m-1) \tau)\right)$. Além disso, este trabalho também avalia o uso de bancos de filtros construídos no intervalo de $(4-40) \mathrm{Hz}$ com subbandas $b \in B$ de largura de $4 \mathrm{~Hz}$, que produzem novos sinais $\mathbf{Z}_{b}$ e sobre cada qual calcula-se um filtro $\mathbf{W}_{b}^{*}$ por meio do CSSP ou CSSSP, tal como ilustra a Figura 7.

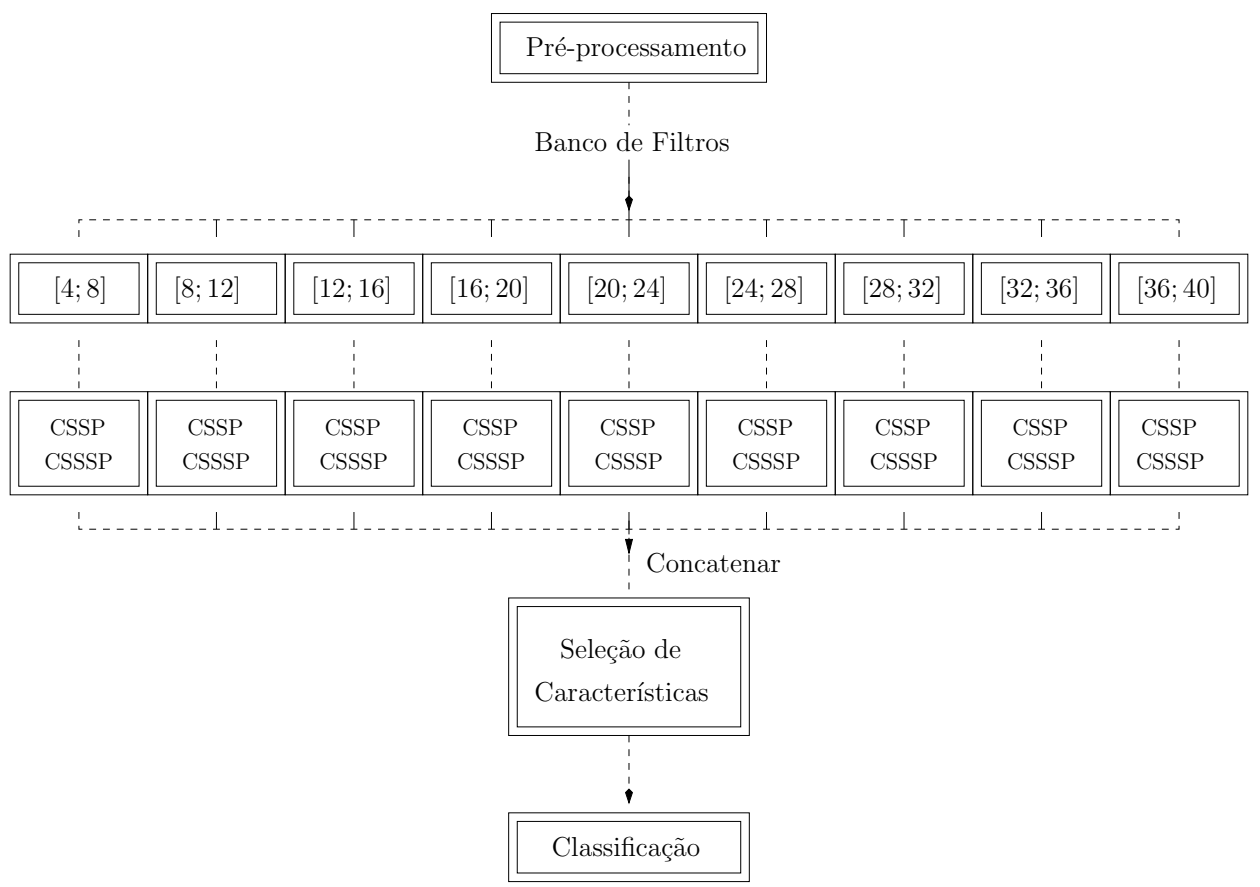

Figura 7. Etapas de classificação de Imagética Motora envolvendo banco de filtros, CSP, CSSP ou CSSSP e seleção de características.

Define-se então, para cada uma das técnicas, CSSP, CSSSP, FBCSSP e FBCSSSP, novos sinais $\mathbf{W}^{*} \mathbf{Z}_{\tau}^{e}, \mathbf{W}^{*} \mathbf{Z}_{m, \tau}^{e}, \mathbf{W}_{b}^{*} \mathbf{Z}_{b, \tau}^{e}$ e $\mathbf{W}_{b}^{*} \mathbf{Z}_{b, m, \tau}^{e}$ para $e \in E$ e $b \in B$, respectivamente. Sobre eles é calculado o logaritmo da variância dos sinais encontrados em cada canal $c \in C$ e suas dimensões embutidas $L=\{l \mid 0 \leq l<m\}$ (e cada sub-banda $b$ se for aplicável) como descritor de características, tal como define a Equação 5. em que $Q=C \times L$ no caso do CSSP e CSSSP e $Q=C \times L \times B$ no caso do FBCSSP e FBCSSSP.

$$
\mathbf{v}^{e}=\left(\log \left(\operatorname{var}\left(\tilde{\mathbf{Z}}_{q}^{e}\right)\right)\right)_{q \in Q} \in \mathcal{V}
$$

Contudo, essas abordagens tendem a produzir um espaço de características de alta dimen- 
Emprego de Banco de Filtros e do Teorema de Imersão de Takens em Padrões Espaciais para a Classificação de Imagética Motora em Interfaces Cérebro-Computador

sionalidade o que motivou, neste trabalho, o emprego da técnica Seleção de Características baseada em Informação Mútua (MIFS) para sua redução. Ainda aplicada sobre o conjunto de sinais de treinamento, a MIFS efetua uma busca gulosa sobre o espaço de características $\mathcal{V}$ adotando o ganho de informação como critério de seleção de atributos tal como apresenta o Algoritmo 1. em que $\tilde{\mathbf{v}}$ é um vetor coluna do espaço $\mathcal{V}, \tilde{\mathbf{y}} \in \mathcal{Y}$ seus respectivos rótulos e $k$ o número de atributos a ser selecionados. Após a seleção de características, adota-se as SVMs com kernel radial para a classificação da Imagética Motora. Todos os processos e técnicas utilizadas por este trabalho são ilustrados na Figura 8
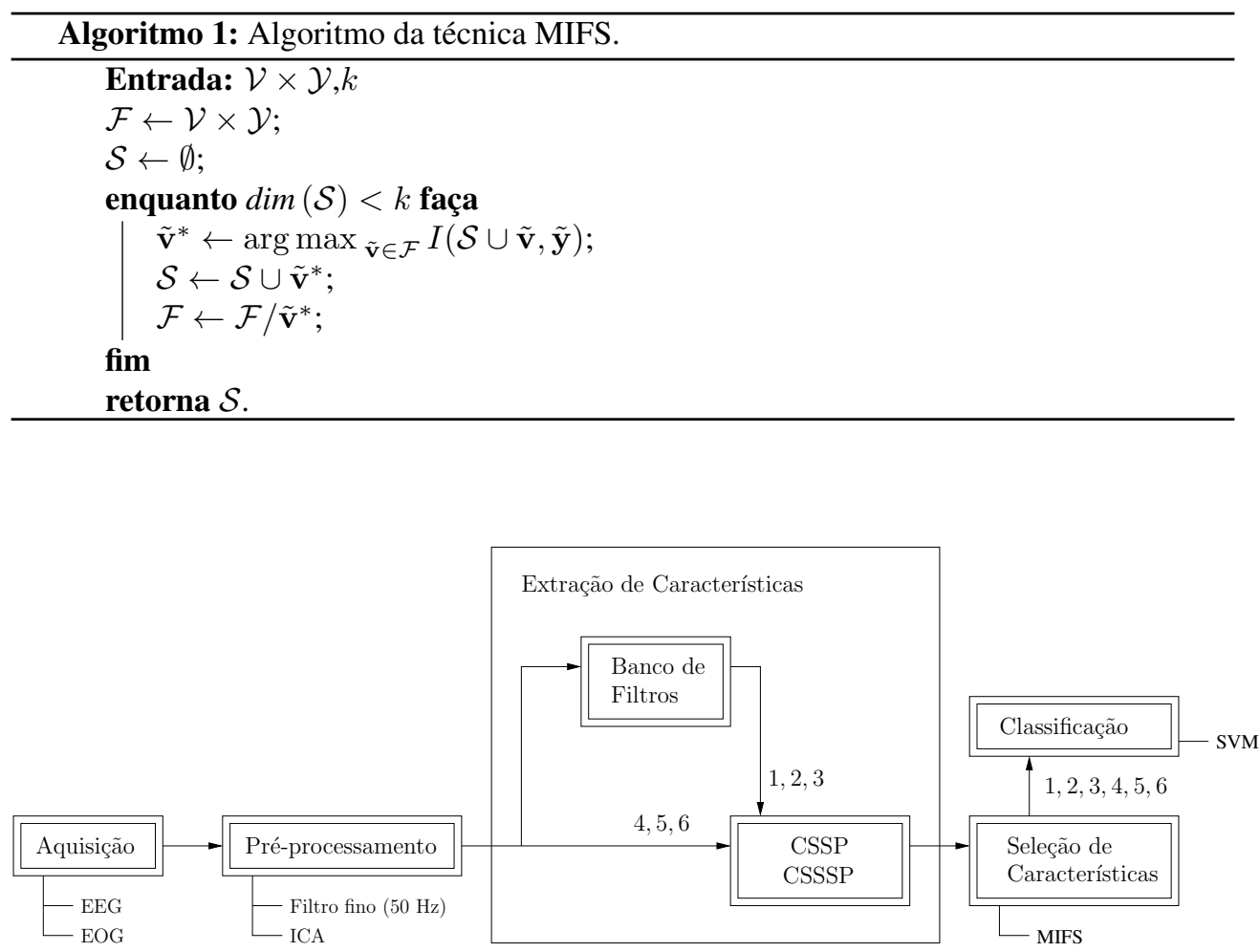

Figura 8. Ilustração das etapas envolvidas para a classificação de sinais de controle em Interfaces Cérebro-Computador e os métodos considerados neste trabalho. Os fluxos 1, $2 \mathrm{e}$ 3 apresentam as abordagens que utilizam banco de filtros e filtros espaciais (CSP, CSSP, CSSSP). Os fluxos 4,5 e 6 apresentam o uso apenas dos filtros espaciais. 
Emprego de Banco de Filtros e do Teorema de Imersão de Takens em Padrões Espaciais para a Classificação de Imagética Motora em Interfaces Cérebro-Computador

\section{Experimentos e Resultados}

Este trabalho conta com um conjunto inicial de experimentos realizados para avaliar o potencial das abordagens propostas, os quais são efetuados sobre diversas configurações de parâmetros (i.e., efetuando uma varredura paramétrica) para o limiar $\theta$ utilizado na identificação de artefatos EOG, para a coordenada de atraso $\tau$, para o número de dimensões embutidas $m$, para o tamanho da janela $t_{f}$ e para a quantidade $k$ de atributos selecionados pela MIFS. Mais precisamente, emprega-se os conjuntos $\Theta=\{\theta \mid \theta=0,1 ; 0,2 ; \ldots ; 1\}$, $\mathcal{M}=\{m \mid m=1 ; 2 ; \ldots ; 10\}, \mathcal{T}=\{\tau \mid \tau=1 ; 2 ; \ldots ; 10\}, \mathcal{J}=\left\{t_{f} \mid t_{f}=\{1 ; 2\}\right\} \mathrm{e}$ $\mathcal{K}=\{k \mid k=1 ; 2 ; \ldots ; \min (30, \operatorname{dim}(\mathcal{V}))\}$ executando tais experimentos sobre o espaço paramétrico $\mathcal{P}_{\tau}=\mathcal{J} \times \mathcal{K} \times \Theta \times \mathcal{T}$ no caso do CSSP e do FBCSSP e $\mathcal{P}_{\tau, m}=\mathcal{J} \times \mathcal{K} \times \Theta \times \mathcal{T} \times \mathcal{M}$ no caso do CSSSP e FBCSSSP.

A execução desse primeiro conjunto de experimentos contou com etapas de treinamento, efetuadas sobre as sessões experimentais 1T, 2T e 3T, e de teste, efetuadas sobre as sessões $4 \mathrm{E}$ e $5 \mathrm{E}$ (maiores detalhes na Seção 2). Essas etapas empregaram cada um dos conjuntos de parâmetros dentro do espaço $\mathcal{P}$, produzindo, por meio de cada teste, seu referente índice kappa. Selecionou-se, então, aquele que apresentou melhores resultados.

Comparando os resultados do CSSP, CSSSP, FBCSSP e FBCSSSP com os das abordagens que compõe o estado da arte, tal como apresenta a Tabela 1 , nota-se que o CSSSP e FBCSSSP podem apresentar índices kappa médios maiores que aqueles do estado da arte. Contudo, aplicando-se o teste de Wilcoxon [34] bilateral com confiança de 0,95, não há diferenças estatisticamente significativas entre as técnicas apresentadas na Tabela 1 ( $p$-valor $>0,025)$. Portanto, o CSSP, CSSSP, FBCSSP e FBCSSSP podem produzir resultados equiparáveis aos do estado da arte.

Tabela 1. Tabela com os índices kappa dos métodos que compõe o estado da arte e das abordagens propostas neste trabalho $\left({ }^{1}[4],{ }^{2}[25]\right.$ e $\left.{ }^{3}[24]\right)$.

\begin{tabular}{|c|c|c|c|c|c|c|c|c|c|c|c|}
\hline & \multicolumn{9}{|c|}{ Voluntários } & \multirow[b]{2}{*}{ Média } & \\
\hline $\begin{array}{c}\text { Método } \\
\text { Autor }\end{array}$ & 1 & 2 & 3 & 4 & 5 & 6 & 7 & 8 & 9 & & $\begin{array}{l}\text { Desvio } \\
\text { Padrão }\end{array}$ \\
\hline CSSP & 0,46 & 0,39 & 0,49 & 0,88 & 0,5 & 0,53 & 0,78 & 0,83 & 0,6 & 0,61 & 0,18 \\
\hline FBCSSP & 0,60 & 0,38 & 0,62 & 0,90 & 0,45 & 0,58 & 0,78 & 0,85 & 0,64 & 0,64 & 0,17 \\
\hline CSSSP & 0,61 & 0,41 & 0,68 & 0,97 & 0,54 & 0,74 & 0,83 & 0,88 & 0,71 & 0,71 & 0,17 \\
\hline FBCSSSP & 0,65 & 0,45 & 0,69 & 0,97 & 0,48 & 0,74 & $\mathbf{0 , 8 4}$ & 0,86 & 0,71 & $\mathbf{0 , 7 1}$ & 0,17 \\
\hline Chin $^{1}$ & 0,40 & 0,21 & 0,22 & 0,95 & 0,86 & 0,61 & 0,56 & 0,85 & 0,74 & 0,60 & 0,28 \\
\hline Gaur $^{2}$ & 0,26 & 0,36 & 0,96 & 0,76 & 0,94 & 0,52 & 0,46 & 0,76 & 0,70 & 0,62 & 0,25 \\
\hline $\mathrm{Saa}^{3}$ & 0,60 & 0,32 & 0,06 & 0,97 & 0,87 & 0,78 & 0,63 & 0,88 & $\mathbf{0 , 8 1}$ & 0,66 & 0,30 \\
\hline
\end{tabular}

Contudo, cabe ressaltar que este experimento não considera a distribuição de probabilidades dos índices kappa produzidos por estas abordagens e, portanto, não retrata fielmente suas capacidades de generalização. Para complementar, os experimentos preliminares efetu- 
Emprego de Banco de Filtros e do Teorema de Imersão de Takens em Padrões Espaciais para a Classificação de Imagética Motora em Interfaces Cérebro-Computador

ados neste trabalho exigem alto custo computacional de forma que a pré-seleção automática dos parametros $\theta, \tau$ e $m$ viabiliza a execução de testes de estabilidade, tal como a validação cruzada de $k$-folds, e, além do mais, considera um cenário mais próximo da aplicação das BCIs.

Neste contexto, avaliou-se, para o CSSP, CSSSP, FBCSSP e FBCSSSP, o comportamento dos índices kappa produzidos para cada índividuo em relação aos limiares $\theta$ adotados no experimento. Contudo, deve-se evitar a influência de grandes variações dos valores de kappa dentre os resultados de cada voluntário. Empregou-se, então, a normalização dos índices kappa de cada voluntário pelo seu máximo valor produzido por um determinado limiar $\theta$, tal como apresenta a Equação 6 em que $i$ é o índice do indivíduo e $\boldsymbol{\kappa}_{i}$ corresponde a um vetor com os valores de kappa associados a cada limiar $\theta$.

$$
\mathbf{v}_{i}=\frac{\boldsymbol{\kappa}_{i}}{\max \left(\boldsymbol{\kappa}_{i}\right)}
$$

Posteriormente, estimou-se o vetor de médias $\overline{\mathbf{v}}$ dentre os indivíduos, o qual foi calculado a partir de $\overline{\mathbf{v}}=\sum_{i=1}^{9} \mathbf{v}_{i}$. A Figura 9 apresenta os resultados de cada elemento de $\overline{\mathbf{v}}$ associado a um limiar $\theta \in \Theta$, indicando que os melhores são produzidos quando nenhum IC é desconsiderado pela ICA no processo de remoção de artefatos. Conclui-se, portanto, que o CSSP, CSSSP, FBCSSP e FBCSSSP são robustos a esses tipos de ruídos. Cabe ressaltar então, que estas técnicas, ao otimizar simultaneamente as variâncias de sinais de controle relacionados às classes de Imagética Motora, tendem, também, a reduzir a influência desses artefatos por meio da transformação linear $\mathbf{W}^{*}$. Assim, nos experimentos seguintes, é desconsiderado o uso da ICA na fase de pré-processamento.

Ademais, foi utilizada para o cálculo dos autovetores generalizado, a função DGGEV do pacote LAPACK, a qual apresenta complexidade assintótica de $\mathcal{O}\left(n^{3}\right)$. Neste sentido, este procedimento produz um gargalo no tempo de execução do treinamento das abordagens empregadas neste trabalho. Adotou-se então, uma pré-seleção automática das coordenadas de atraso $\tau$ e do número de dimensões embutidas $m$, baseadas nos trabalhos de [35] e [36], os quais consideram, respectivamente, a Auto-Informação Mútua (do inglês Auto Mutual Information - AMI) para se estimar $\tau$ e a técnica de Falsos Próximos Vizinhos (do inglês False Nearest Neighbors - FNN) para se estimar $m$.

A AMI permite encontrar um valor adequado para a coordenada de atraso $\tau$, segundo um critério de recorrência temporal dos dados. Mais precisamente, essa equação calcula o ganho de informação que a série $z_{c}^{e} \in \mathbf{Z}^{e}$, quando dada como entrada, provê em relação a um tempo futuro $\tau$. Seleciona-se, tipicamente, o instante de tempo $\tau$ referente ao primeiro mínimo de $\left\{\operatorname{AMI}\left(z_{c}, z_{c, \tau}\right): \tau=\left[1, \tau_{f}\right]\right\}$ como coordenada de atraso. Isso se deve ao fato de que esse mínimo oferece o primeiro menor ganho de informação, o qual auxilia a desdobrar de maneira mais adequada os dados no espaço fase [35], segundo o Teorema de Imersão 
Emprego de Banco de Filtros e do Teorema de Imersão de Takens em Padrões Espaciais para a Classificação de Imagética Motora em Interfaces Cérebro-Computador

Método: $\rightarrow$ CSSP - CSSSP - F FBCSSP + FBCSSSP

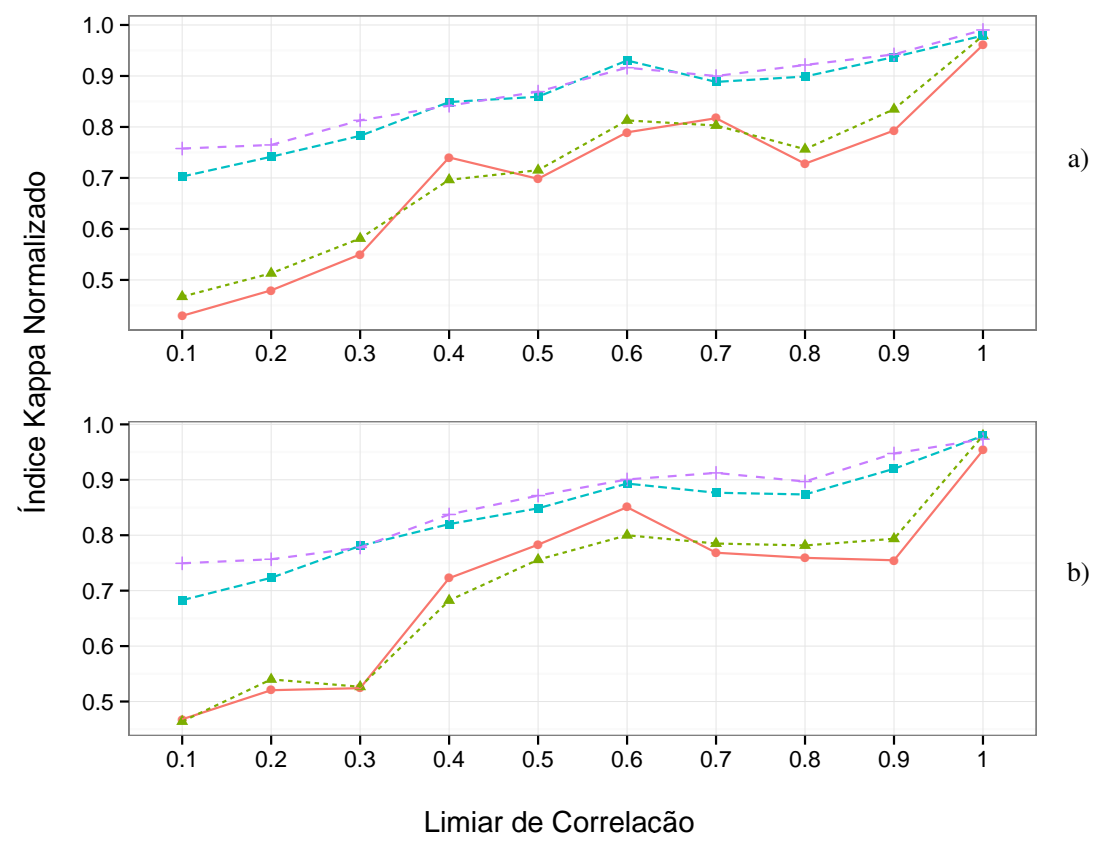

Figura 9. Gráficos dos índices kappa normalizados médios vs. limiares de correlação para remoção de artefatos. Esses dados foram produzidos a partir da aplicação dos métodos das abordagens avaliadas sobre janelas de: a) um segundo e b) dois segundos extraídos dos períodos de Imagética Motora.

proposto por [23]. Caso não haja um primeiro mínimo bem destacado, a literatura sugere o uso do atraso $\tau=1$ [37].

Cabe ressaltar que os dados abordados neste trabalho são formados por diversas séries temporais (sinais) contidos em diferentes ensaios $e \in E$ e canais $c \in C$. Portanto, tal como ilustra a Figura 10, estima-se uma coordenada de atraso $\tau_{c}$ que seja adequada a apenas um canal $c$ e a todos ensaios calculando, primeiramente, as coordenadas de atraso $\tau_{c}^{e}$ associadas a cada canal $c$ contidos em cada ensaio $e$. Posteriormente, para cada $c$, a mediana é aplicada 
Emprego de Banco de Filtros e do Teorema de Imersão de Takens em Padrões Espaciais para a Classificação de Imagética Motora em Interfaces Cérebro-Computador

ao longo de todos os ensaios $e$, produzindo um conjunto $\mathcal{T}$ de coordenadas de atraso $\tau_{c}$ que são adotadas em seus respectivos canais ao se empregar o Teorema de Imersão de Takens [23].

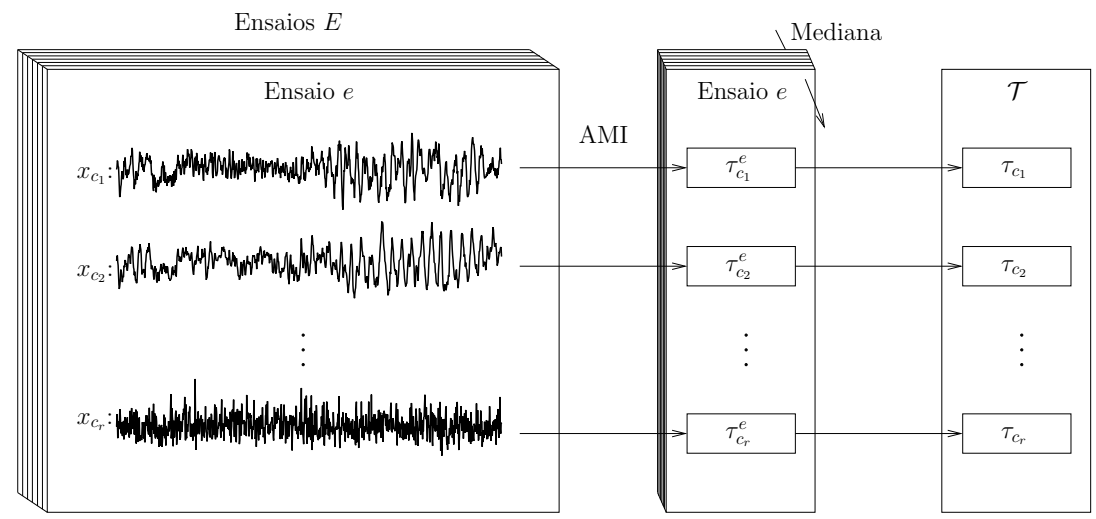

Figura 10. Fluxo de dados para estimar as coordenadas de atraso dos sinais de controle produzidos em cada canal $c_{j}$.

Em complemento, foi empregada a técnica de Falsos Vizinhos mais Próximos (do inglês False Nearest Neighbors - FNN) para a estimação do número de dimensões embutidas $m_{c}^{e}$ dos sinais associados a cada ensaio $e$ e canal $c$, avaliando se imersões subsequentes de um espaço fase $z_{c, m-1, \tau}^{e}(t)$ e em um outro $z_{c, m, \tau}(t)$ produz distorções [36]. Tal como ilustra a Figura 11, foram calculados os números de dimensões embutidas $m_{c} \in \mathcal{M}$ que melhor se adequassem a cada canal $c$, para os quais foi aplicada a mediana dos conjuntos $\mathcal{M}_{c}=$ $\left\{m_{c}^{e} \mid \forall e \in E\right\}$, i.e., ao longo de todos ensaios $e$. Cabe ressaltar que, quando empregados os bancos de filtros em conjunto com o CSSP e CSSSP, $\tau$ e $m$ são estimados não mais para cada canal $c$, e sim para cada par ordenado $(c, b) \in C \times B$ que compõe as combinações entre sub-bandas $b$ e canais $c$.

Estimando-se automaticamente as coordenadas de atraso e dimensões embutidas, reduzse o custo computacional despendido na etapa de configuração desses parâmetros para um indivíduo específico. Esta redução permitiu a realização de experimentos adicionais utilizando a técnica de validação cruzada de 10 folds [38] que permite a estimação da estabilidade de um algoritmo de aprendizado de máquina. Em conjunto com o índice kappa, foi considerado o ganho de informação que cada dimensão selecionada pelo MIFS (a partir do espaço fase obtido) produz para melhor avaliar quais números de dimensões $k$.

Como exemplificado na Figura 12 os resultados dos índices kappa indicam a possibilidade de definir um critério de parada para o algoritmo MIFS em função da convergência 
Emprego de Banco de Filtros e do Teorema de Imersão de Takens em Padrões Espaciais para a Classificação de Imagética Motora em Interfaces Cérebro-Computador

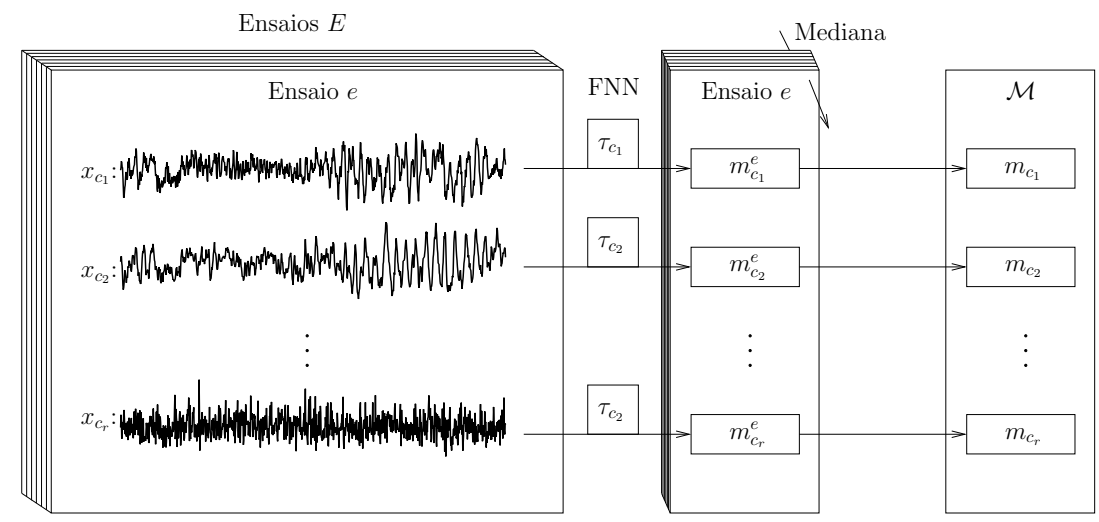

Figura 11. Fluxo de dados para estimar o número de dimensões embutidas dos sinais de controle produzidos em cada canal $c_{j}$.

do ganho de informação. Neste trabalho, essa convergência é detectada quando a diferença absoluta entre o ganho de informação produzido após a adição de uma nova dimensão atinge um limiar menor que $1 \times 10^{-5}$. Cabe ressaltar que os experimentos apresentados a seguir foram efetuados a partir das abordagens de estimação de parâmetros: i) $m_{c}$ para a dimensão embutida para cada canal $c$; ii) $\tau_{c}$ para a coordenada de atraso de cada canal $c$; e, finalmente, iii) $k$ para definir o número de dimensões do espaço fase selecionadas pelo algoritmo MIFS.

Considerando a validação cruzada de 10 folds, cujos resultados são apresentados nas Figuras $13 \mathrm{e} 14$, a parametrização automática produz melhores índices kappa para determinados indivíduos quando comparados à varredura de todo o espaço paramétrico, tal como abordado nos experimentos preliminares. Sob uma perspectiva estatística, o uso da abordagem automática para a estimação das coordenadas de atraso, dimensões embutidas e dimensões selecionadas pelo algoritmo MIFS é justificável uma vez que reduz o custo computacional da configuração dos extratores e da seleção de características.

Contudo, utilizando as sessões 1T,2T e $3 \mathrm{~T}$ para treinamento e as $4 \mathrm{E}$ e $5 \mathrm{E}$ para teste, a Tabela 2 indica que essa parametrização automática produz resultados inferiores ao estado da arte e distantes da configuração ótima (avaliando todo o espaço paramétrico). Em contrapartida, cabe ressaltar que a escolha do número $k$ de dimensões selecionadas pelo MIFS por meio da convergência de ganho de informação produz resultados abaixo do esperado. Como apresenta a Tabela 3 , ao selecionar o melhor $k$ dentro do espaço paramétrico $\mathcal{K}$, os índices kappa do CSSP, CSSSP, FBCSSP e FBCSSSP aumentam consideravelmente. 
Emprego de Banco de Filtros e do Teorema de Imersão de Takens em Padrões Espaciais para a Classificação de Imagética Motora em Interfaces Cérebro-Computador

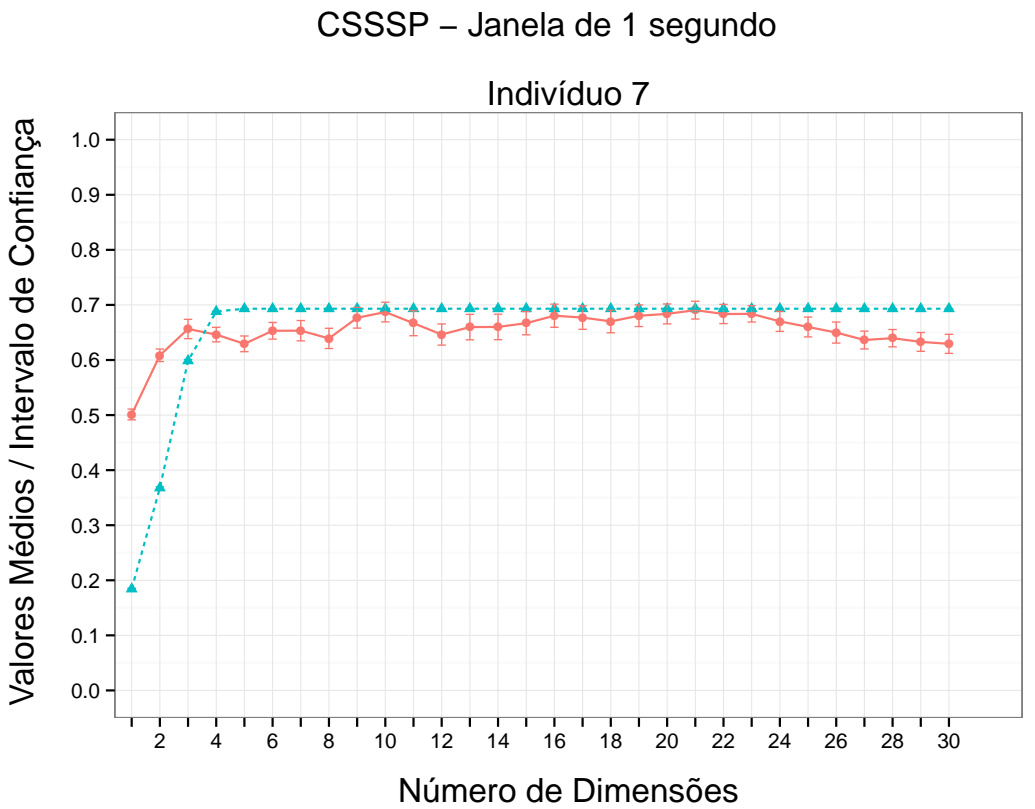

Métrica: $\rightarrow$ Índice Kappa - Informação Mútua

Figura 12. Gráfico ilustrando a convergência do ganho de informação, produzido a partir da validação cruzada 10 fold executada sobre os sinais de EEG do indivíduo 7, utilizando o CSSSP para extração de características e parametrização automática.

\section{Conclusão}

A partir do primeiro conjunto de experimentos foi possível concluir que as técnicas CSSSP e FBCSSSP podem atingir resultados equiparáveis ao estado da arte, contudo, uma série de parâmetros deve ser definidos para: i) remoção de artefatos $(\theta)$; ii) imersão dos sinais $(\tau$ e $m)$; e iii) número de atributos selecionados $(k)$. De acordo com esses primeiros resultados experimentais, conclui-se que as abordagens de extração de características empregadas neste trabalho (CSSP, CSSSP, FBCSSP e FBCSSSP) são robustas a influências de artefatos, uma vez que: i) por suas formulações, há a produção de um modelo autorregressivo capaz de 
Emprego de Banco de Filtros e do Teorema de Imersão de Takens em Padrões Espaciais para a Classificação de Imagética Motora em Interfaces Cérebro-Computador

Método: $\rightarrow$ Melhor Configuração $\rightarrow$ Parametrização Automática

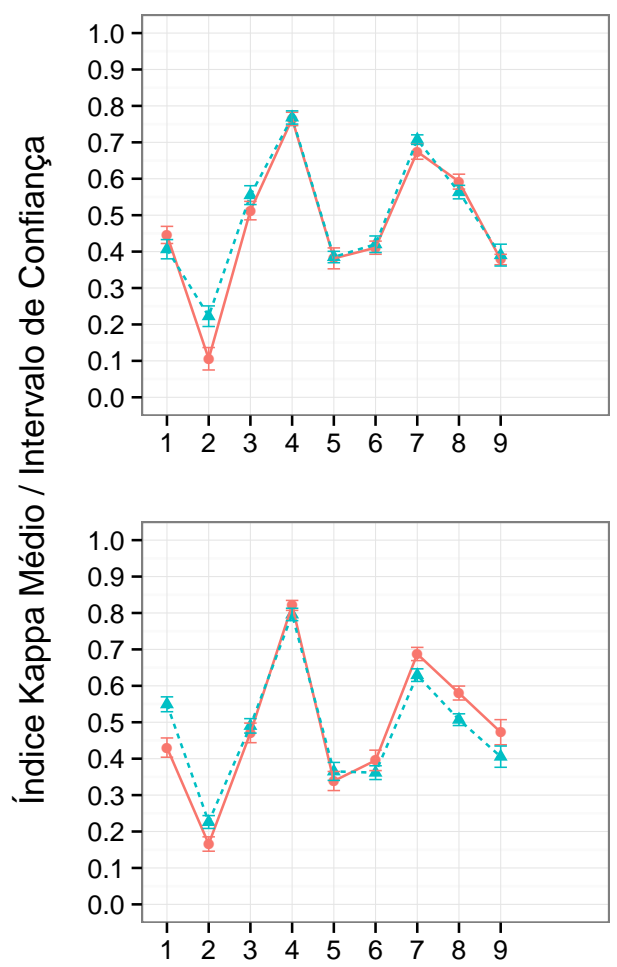

a)
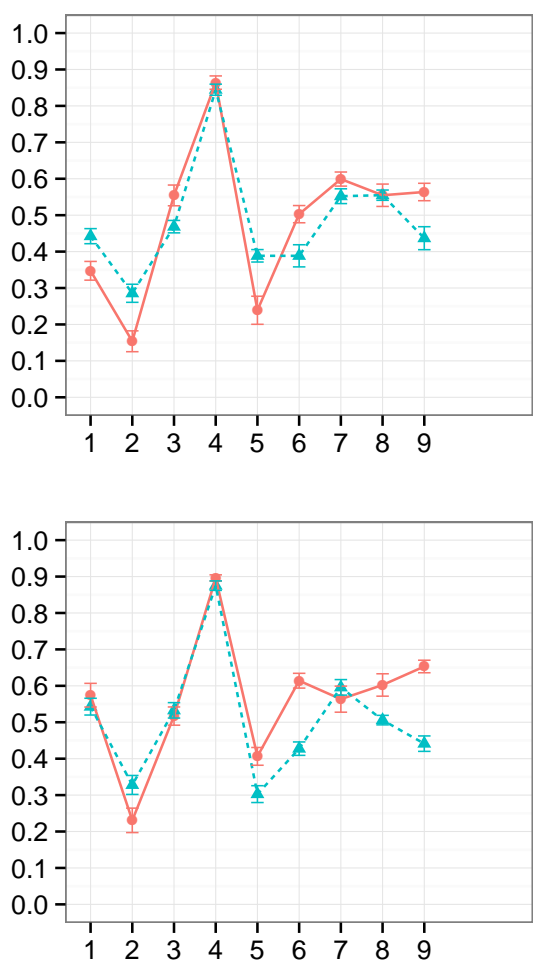

b)

Indivíduo

Figura 13. Gráficos dos índices kappa Médios Intervalo de Confiança vs. Indivíduo considerando as abordagens de [35] e [36] para a encontrar coordenadas de atraso e dimensões embutidas para o CSSP e CSSSP e os parâmetros que resultaram em melhores acurácia nos experimentos preliminares. Esses resultados foram produzidos sobre janelas de: a) um segundo e b) dois segundos extraídos dos períodos de Imagética Motora.

mitigar as influências de Eletrooculografia quando os canais de EOG são inclusos no cáuclo de autovetores generalizados; e ii) a decomposição em sub-bandas de frequência por meio de um banco de filtros permite a eliminação de artefatos na etapa de seleção de característi- 
Emprego de Banco de Filtros e do Teorema de Imersão de Takens em Padrões Espaciais para a Classificação de Imagética Motora em Interfaces Cérebro-Computador

Método: $\rightarrow$ Melhor Configuração $\rightarrow$ Parametrização Automática

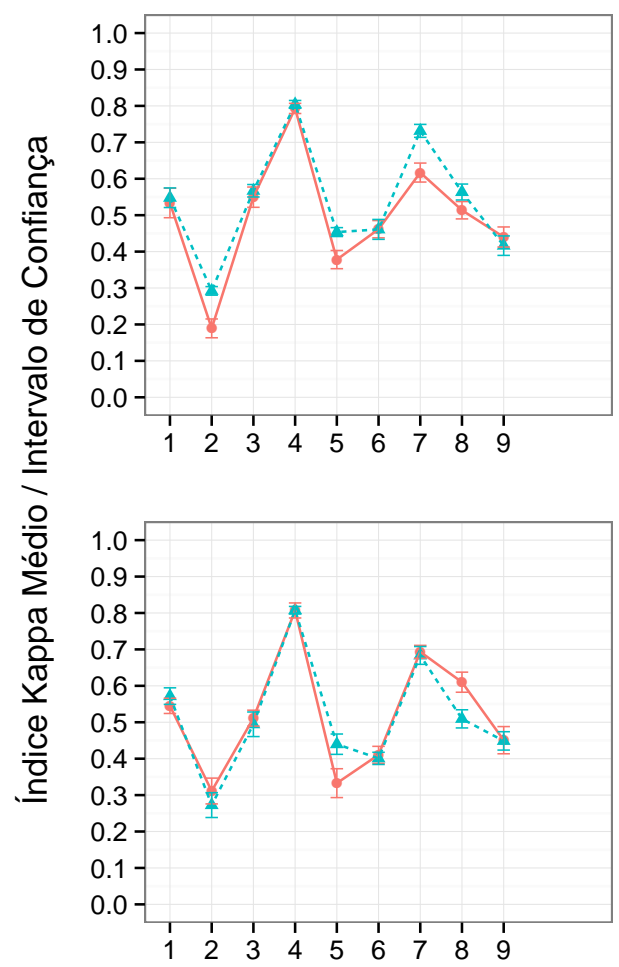

a)
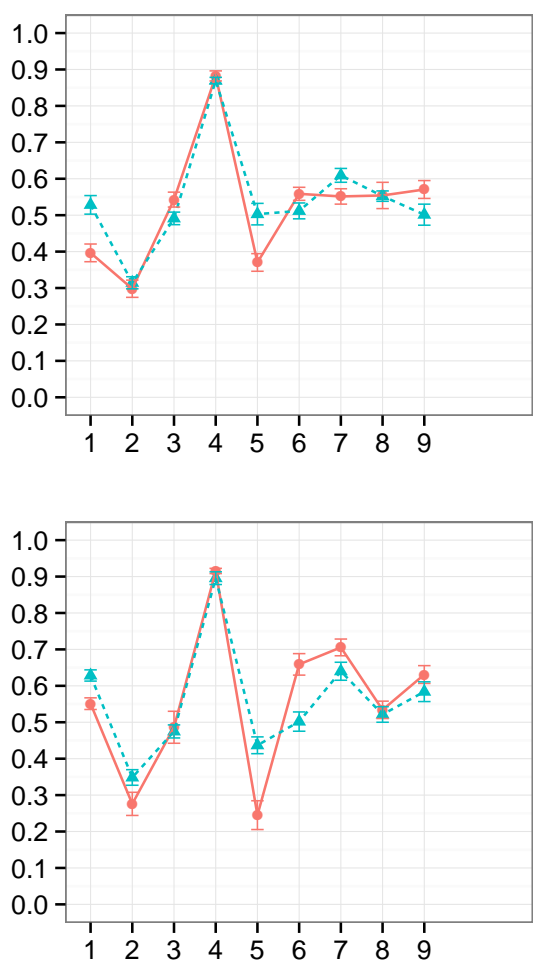

b)

Indivíduo

Figura 14. Gráficos dos índices kappa Médios Intervalo de Confiança vs. Indivíduo considerando as abordagens de [35] e [36] para a encontrar coordenadas de atraso e dimensões embutidas para o FBCSSP e FBCSSSP e os parâmetros que resultaram em melhores acurácia nos experimentos preliminares. Esses resultados foram produzidos sobre janelas de: a) um segundo e b) dois segundos extraídos dos períodos de Imagética Motora.

cas, pois esses supostamente não garantem ganho de informação (calculado pelo algoritmo MIFS). 
Emprego de Banco de Filtros e do Teorema de Imersão de Takens em Padrões Espaciais para a Classificação de Imagética Motora em Interfaces Cérebro-Computador

Tabela 2. Tabela com os índices kappa dos métodos utilizando a configuração automática de parâmetros e com resultados do estado da arte $\left({ }^{1}[4],{ }^{2}[25] \mathrm{e}^{3}[24]\right)$.

\begin{tabular}{|c|c|c|c|c|c|c|c|c|c|c|c|}
\hline & \multicolumn{9}{|c|}{ Voluntários } & \multirow[b]{2}{*}{ Média } & \multirow[b]{2}{*}{$\begin{array}{l}\text { Desvio } \\
\text { Padrão }\end{array}$} \\
\hline Janela & 1 & 2 & 3 & 4 & 5 & 6 & 7 & 8 & 9 & & \\
\hline \multicolumn{12}{|c|}{ CSSP } \\
\hline $1 \mathrm{seg}$. & 0,41 & 0,17 & 0,29 & 0,43 & 0,00 & 0,09 & 0,65 & 0,36 & 0,41 & 0,31 & 0,20 \\
\hline 2 seg. & 0,09 & 0,07 & 0,34 & 0,43 & 0,14 & 0,16 & 0,48 & 0,61 & 0,27 & 0,29 & 0,19 \\
\hline Máximo & 0,41 & 0,17 & 0,34 & 0,43 & 0,14 & 0,16 & 0,65 & 0,61 & 0,41 & 0,37 & 0,19 \\
\hline \multicolumn{12}{|c|}{ CSSSP } \\
\hline $1 \mathrm{seg}$. & 0,35 & 0,21 & 0,18 & 0,86 & 0,12 & 0,32 & 0,78 & 0,48 & 0,21 & 0,39 & 0,27 \\
\hline 2 seg. & 0,18 & 0,13 & 0,18 & 0,93 & 0,09 & 0,27 & 0,38 & 0,43 & 0,41 & 0,33 & 0,26 \\
\hline Máximo & 0,35 & 0,21 & 0,18 & 0,93 & 0,12 & 0,32 & 0,78 & 0,48 & 0,41 & 0,42 & 0,27 \\
\hline \multicolumn{12}{|c|}{ FBCSSP } \\
\hline $1 \mathrm{seg}$. & 0,17 & 0,17 & 0,28 & 0,56 & 0,25 & 0,17 & 0,32 & 0,44 & 0,26 & 0,29 & 0,13 \\
\hline 2 seg. & 0,19 & 0,18 & 0,34 & 0,61 & 0,17 & 0,42 & 0,51 & 0,64 & 0,52 & 0,40 & 0,19 \\
\hline Máximo & 0,19 & 0,18 & 0,34 & 0,61 & 0,25 & 0,42 & 0,51 & 0,64 & 0,52 & 0,41 & 0,18 \\
\hline \multicolumn{12}{|c|}{ FBCSSSP } \\
\hline $1 \mathrm{seg}$. & 0,43 & 0,15 & 0,34 & 0,84 & 0,05 & 0,13 & 0,79 & 0,43 & 0,45 & 0,40 & 0,28 \\
\hline 2 seg. & 0,27 & 0,27 & 0,15 & 0,95 & 0,10 & 0,48 & 0,59 & 0,69 & 0,47 & 0,44 & 0,28 \\
\hline Máximo & 0,43 & 0,27 & 0,34 & 0,95 & 0,10 & 0,48 & 0,79 & 0,69 & 0,47 & 0,50 & 0,27 \\
\hline \multicolumn{12}{|c|}{ Estado da arte } \\
\hline Chin $^{1}$ & 0,40 & 0,21 & 0,22 & 0,95 & 0,86 & 0,61 & 0,56 & 0,85 & 0,74 & 0,60 & 0,28 \\
\hline Gaur $^{2}$ & 0,26 & 0,36 & 0,96 & 0,76 & 0,94 & 0,52 & 0,46 & 0,76 & 0,70 & 0,62 & 0,25 \\
\hline $\mathrm{Saa}^{3}$ & 0,60 & 0,32 & 0,06 & 0,97 & 0,87 & 0,78 & 0,63 & $\mathbf{0 , 8 8}$ & $\mathbf{0 , 8 1}$ & 0,66 & 0,30 \\
\hline
\end{tabular}

Em complemento, a varredura de todo o espaço paramétrico para a seleção das coordenadas de atraso, dimensões embutidas e atributos selecionadas pelo MIFS é uma tarefa de alto custo computacional. Portanto, adotou-se as técnicas de Falsos Vizinhos mais Próximos (do inglês False Nearest Neighbors - FNN) [36] e Auto-Informação Mútua (do inglês Auto-Mutual Information - AMI) [35] para estimar a quantidade de dimensões embutidas e as coordenadas de atraso mais adequadas, respectivamente, para produzir a imersão dos sinais de controle [23]. Essas técnicas selecionam os parâmetros mencionados por meio de critérios reconhecidos na literatura [37]. Avaliou-se, ainda, a convergência do ganho de informação calculado pelo algoritmo MIFS como critério para definir o número de dimensões utilizado pelo o conjunto de dados empregado na etapa de classificação.

A parametrização automática não implicou em maiores índices kappa quando executados os experimentos de classificação utilizando o mesmo método considerado pela $B C I$ Competition IV-2b. Contudo, essa automatização permitiu a execução de experimentos adicionais a fim de avaliar a capacidade de generalização dos classificadores obtidos, uma vez que reduziu significativamente o tempo de processamento despendido. Essa etapa considerou o método de validação cruzada de 10 folds para verificar o comportamento das abordagens de extração e seleção de características. Concluiu-se que os índices kappa obtidos a partir da parametrização automática são equiparáveis aos produzidos pelo melhor cenário da varredura paramétrica. Logo, essa automatização é util como ferramental para a etapa de configuração 
Emprego de Banco de Filtros e do Teorema de Imersão de Takens em Padrões Espaciais para a Classificação de Imagética Motora em Interfaces Cérebro-Computador

Tabela 3. Tabela com os índices kappa dos métodos utilizando a configuração automática dos parâmetros $\tau$ e $m$ (adotando-se o parâmetro $k$ que produz melhores resultados) e com resultados do estado da arte $\left({ }^{1}[4],{ }^{2}[25] \mathrm{e}^{3}[24]\right)$.

\begin{tabular}{|c|c|c|c|c|c|c|c|c|c|c|c|}
\hline & \multicolumn{9}{|c|}{ Voluntários } & \multirow[b]{2}{*}{ Média } & \multirow[b]{2}{*}{$\begin{array}{l}\text { Desvio } \\
\text { Padrão }\end{array}$} \\
\hline Janela & 1 & 2 & 3 & 4 & 5 & 6 & 7 & 8 & 9 & & \\
\hline \multicolumn{12}{|c|}{ CSSP } \\
\hline $1 \mathrm{seg}$. & 0,41 & 0,26 & 0,37 & 0,43 & 0,30 & 0,34 & 0,75 & 0,51 & 0,41 & 0,42 & 0,14 \\
\hline 2 seg. & 0,16 & 0,18 & 0,34 & 0,45 & 0,25 & 0,33 & 0,48 & 0,78 & 0,36 & 0,37 & 0,19 \\
\hline Máximo & 0,41 & 0,24 & 0,32 & 0,41 & 0,31 & 0,14 & 0,35 & 0,73 & 0,37 & 0,45 & 0,19 \\
\hline \multicolumn{12}{|c|}{ CSSSP } \\
\hline $1 \mathrm{seg}$. & 0,46 & 0,27 & 0,42 & 0,86 & 0,16 & 0,42 & 0,78 & 0,63 & 0,38 & 0,49 & 0,23 \\
\hline 2 seg. & 0,36 & 0,23 & 0,40 & 0,94 & 0,14 & 0,42 & 0,60 & 0,68 & 0,57 & 0,48 & 0,24 \\
\hline Máximo & 0,46 & 0,27 & 0,42 & 0,94 & 0,16 & 0,42 & 0,78 & 0,68 & 0,57 & 0,52 & 0,25 \\
\hline \multicolumn{12}{|c|}{ FBCSSP } \\
\hline $1 \mathrm{seg}$. & 0,29 & 0,27 & 0,47 & 0,66 & 0,37 & 0,27 & 0,45 & 0,54 & 0,45 & 0,42 & 0,13 \\
\hline $2 \mathrm{seg}$. & 0,24 & 0,26 & 0,35 & 0,73 & 0,37 & 0,43 & 0,58 & 0,73 & 0,61 & 0,48 & 0,19 \\
\hline Máximo & 0,29 & 0,27 & 0,47 & 0,73 & 0,37 & 0,43 & 0,58 & 0,73 & 0,61 & 0,50 & 0,17 \\
\hline \multicolumn{12}{|c|}{ FBCSSSP } \\
\hline $1 \mathrm{seg}$. & 0,51 & 0,27 & 0,48 & 0,86 & 0,15 & 0,18 & 0,82 & 0,60 & 0,49 & 0,48 & 0,25 \\
\hline 2 seg. & 0,40 & 0,36 & 0,31 & 0,96 & 0,18 & 0,54 & 0,77 & 0,76 & 0,50 & 0,53 & 0,28 \\
\hline Máximo & 0,51 & 0,36 & 0,48 & 0,96 & 0,18 & 0,54 & 0,82 & 0,76 & 0,50 & 0,57 & 0,24 \\
\hline \multicolumn{12}{|c|}{ Estado da arte } \\
\hline Chin $^{1}$ & 0,40 & 0,21 & 0,22 & 0,95 & 0,86 & 0,61 & 0,56 & 0,85 & 0,74 & 0,60 & 0,28 \\
\hline Gaur $^{2}$ & 0,26 & 0,36 & 0,96 & 0,76 & 0,94 & 0,52 & 0,46 & 0,76 & 0,70 & 0,62 & 0,25 \\
\hline $\mathrm{Saa}^{3}$ & 0,60 & 0,32 & 0,06 & 0,97 & 0,87 & 0,78 & 0,63 & $\mathbf{0}, \mathbf{8 8}$ & $\mathbf{0}, \mathbf{8 1}$ & 0,66 & 0,30 \\
\hline
\end{tabular}

de um sistema de BCI para quaisquer indivíduos, sendo fundamental para cenários reais.

\section{Agradecimentos}

Ao Conselho Nacional de Desenvolvimento Científico e Tecnológico (CNPq) - Processo 132118/2014-9.

\section{Contribuição dos autores:}

$\mathrm{O}$ artigo foi redigido a partir do trabalho de mestrado de Yule Vaz, orientado pelo Prof. Dr. Rodrigo Fernandes de Mello. O contexto teórico deste trabalho foi estudado e desenvolvido por ambos pesquisadores. Após sua formulação, Yule Vaz executou os experimentos e seus resultados foram analisados por ambos autores. 
Emprego de Banco de Filtros e do Teorema de Imersão de Takens em Padrões Espaciais para a Classificação de Imagética Motora em Interfaces Cérebro-Computador

\section{Referências}

[1] D. Purves, D. Fitzpatrick, L.C. Katz, A.S. Lamantia, J.O. McNamara, S.M. Williams, and G.J. Augustine. Neuroscience. Sinauer Associates, Sunderland, MA, Estados Unidoos, 2001.

[2] Luis Fernando Nicolas-Alonso and Jaime Gomez-Gil. Brain Computer Interfaces, a Review. Sensors, 12:1211-1279, January 2012.

[3] Bernhard Graimann, Brendan Allison, and Gert Pfurtscheller. Brain-computer interfaces: A gentle introduction. In Bernhard Graimann, Brendan Allison, and Gert Pfurtscheller, editors, Brain-Computer Interfaces: Revolutionizing Human-Computer Interaction. Springer-Verlag Berlin Heidelberg, Tiergartenstreße, Heidelberg, Alemanha, 2010.

[4] Michael Tangermann, Klaus-Robert Müller, Ad Aertsen, Niels Birbaumer, Christoph Braun, Clemens Brunner, Robert Leeb, Carsten Mehring, Kai J Miller, Gernot MuellerPutz, Guido Nolte, Gert Pfurtscheller, Hubert Preissl, Gerwin Schalk, Alois Schlögl, Carmen Vidaurre, Stephan Waldert, and Benjamin Blankertz. Review of the BCI competition IV. Frontiers in Neuroscience, 6(55), 2012.

[5] Jonathan R. Wolpaw, Niels Birbaumer, Dennis J. McFarland, Gert Pfurtscheller, and Theresa M. Vaughan. Brain-computer interfaces for communication and control. Clinical Neurophysiology, 113(6):767-791, June 2002.

[6] Hai Thanh Nguyen, Hoa Van Nguyen, Khoa Quang Dang Truong, and Toi Van Vo. Analysis of oxy-hb signals to determine relationship between jaw imbalance and arm strength using fnirs. American Journal of Biomedical Engineering, 3(5):107-118, 2013.

[7] M. Teplan. Fundamentals of eeg measurement. Measurement Science Review, 2:1-11, 2002.

[8] Mehrdad Fatourechi, Ali Bashashati, Rabab K. Ward, and Gary E. Birch. EMG and EOG artifacts in brain computer interface systems: A survey. Clinical Neurophysiology, 118(3):480-494, 2006.

[9] C. J. Ochoa and J. Polich. P300 and blink instructions. Clin Neurophysiol, 111(1):9398, January 2000.

[10] R. Verleger. The instruction to refrain from blinking affects auditory P3 and N1 amplitudes. Electroencephalogr Clin Neurophysiol, 78(3):240-251, March 1991. 
Emprego de Banco de Filtros e do Teorema de Imersão de Takens em Padrões Espaciais para a Classificação de Imagética Motora em Interfaces Cérebro-Computador

[11] Arthur Flexer, Herbert Bauer, Jürgen Pripfl, and Georg Dorffner. Using ICA for removal of ocular artifacts in EEG recorded from blind subjects. Neural Networks, 18(7):998 $1005,2005$.

[12] Kai Keng Ang, Zheng Yang Chin, Chuanchu Wang, Cuntai Guan, and Haihong Zhang. Filter bank common spatial pattern algorithm on bci competition iv datasets $2 \mathrm{a}$ and $2 \mathrm{~b}$. Frontiers in Neuroscience, 6(39), 2012.

[13] M. Doppelmayr, W. Klimesch, T. Pachinger, and B. Ripper. Individual differences in brain dynamics: important implications for the calculation of event-related band power. Biol Cybern, 79(1):49-57, July 1998.

[14] F. H. Lopes da Silva and G. Pfurtscheller. Basic concepts on EEG synchronization and desynchronization. Thrombosis and Haemostasis, pages 3-11, 1999.

[15] C. Neuper and G. Pfurtscheller. Event-related dynamics of cortical rhythms: frequencyspecific features and functional correlates. Int J Psychophysiol, 43(1):41-58, December 2001.

[16] J. del R Millan, J. Mourino, M. Franze, F. Cincotti, M. Varsta, J. Heikkonen, and F. Babiloni. A local neural classifier for the recognition of EEG patterns associated to mental tasks. Neural Networks, IEEE Transactions on, 13(3):678-686, May 2002.

[17] Haider Raza, Hubert Cecotti, Yuhua Li, and Girijesh Prasad. Adaptive learning with covariate shift-detection for motor imagery-based brain-computer interface. Soft Computing, pages 1-12, 2015.

[18] N.F. Ince, A.H. Tewfik, and S. Arica. Extraction subject-specific motor imagery timefrequency patterns for single trial EEG classification. Computers in Biology and Medicine, 37(4):499-508, 2007.

[19] Z. Zhou and B. Wan. Wavelet packet-based Independent Component Analysis for feature extraction from motor imagery EEG of complex movements. Clinical Neurophysiology, 123(9):1779-1788, 2012.

[20] Z.J. Koles. The quantitative extraction and topographic mapping of the abnormal components in the clinical eeg. Electroencephalography and Clinical Neurophysiology, 79(6):440 - 447, 1991.

[21] S. Lemm, B. Blankertz, G. Curio, and K. R. Muller. Spatio-spectral filters for improving the classification of single trial EEG. IEEE Trans Biomed Eng, 52(9):1541-1548, September 2005. 
Emprego de Banco de Filtros e do Teorema de Imersão de Takens em Padrões Espaciais para a Classificação de Imagética Motora em Interfaces Cérebro-Computador

[22] G. Dornhege, B. Blankertz, M. Krauledat, F. Losch, G. Curio, and K.-R. Muller. Combined optimization of spatial and temporal filters for improving brain-computer interfacing. Biomedical Engineering, IEEE Transactions on, 53(11):2274-2281, November 2006.

[23] Floris Takens. Detecting strange attractors in turbulence. In David Rand and LaiSang Young, editors, Dynamical Systems and Turbulence, Warwick 1980, volume 898 of Lecture Notes in Mathematics, pages 366-381. Springer Berlin Heidelberg, 1981.

[24] J. F. Saa and M. Cetin. A latent discriminative model-based approach for classification of imaginary motor tasks from EEG data. J Neural Eng, 9(2):026020, April 2012.

[25] P. Gaur, R. B. Pachori, Hui Wang, and G. Prasad. An empirical mode decomposition based filtering method for classification of motor-imagery eeg signals for enhancing brain-computer interface. In Neural Networks (IJCNN), 2015 International Joint Conference on, pages 1-7, July 2015.

[26] Bo Hjorth. EEG analysis based on time domain properties. Electroencephalography and Clinical Neurophysiology, 29(3):306 - 310, 1970.

[27] G. Hughes. On the mean accuracy of statistical pattern recognizers. Information Theory, IEEE Transactions on, 14(1):55-63, January 1968.

[28] John Hopcroft and Ravindran Kannan. Foundations of Data Science. 2014.

[29] Corinna Cortes and Vladimir Vapnik. Support-Vector Networks. In Machine Learning, pages 273-297, 1995.

[30] Vladimir N. Vapnik. The Nature of Statistical Learning Theory. Springer-Verlag New York, Inc., New York, NY, Estados Unidos, 1995.

[31] Ulrike von Luxburg and Bernhard Scholkopf. Statistical Learning Theory: Models, concepts and results. Handbook of the History of Logic, 10:651-706, 2009.

[32] J. Cohen. A Coefficient of Agreement for Nominal Scales. Educational and Psychological Measurement, 20(1):37, 1960.

[33] James V. Stone. Independent Component Analysis: A Tutorial Introduction. MIT Press, Cambridge, MA, Estados Unidos, 1999.

[34] Frank Wilcoxon. Individual Comparisons by Ranking Methods. Biometrics Bulletin, 1(6):80-83, December 1945.

[35] Andrew M. Fraser and Harry L. Swinney. Independent coordinates for strange attractors from mutual information. Phys. Rev. A, 33:1134-1140, February 1986. 
Emprego de Banco de Filtros e do Teorema de Imersão de Takens em Padrões Espaciais para a Classificação de Imagética Motora em Interfaces Cérebro-Computador

[36] Matthew B. Kennel, Reggie Brown, and Henry D. I. Abarbanel. Determining embedding dimension for phase-space reconstruction using a geometrical construction. Phys. Rev. A, 45:3403-3411, March 1992.

[37] Rodrigo Fernandes de Mello. Sistemas dinâmicos e técnicas inteligentes para a predição de comportamento de processos: uma abordagem para otimização de escalonamento em grades computacionais. PhD thesis, Instituto de Ciências Matemáticas e de Computação, 2009.

[38] Ron Kohavi. A study of cross-validation and bootstrap for accuracy estimation and model selection. In Proceedings of the 14th International Joint Conference on Artificial Intelligence - Volume 2, IJCAI'95, pages 1137-1143, San Francisco, CA, Estados Unidos, 1995. Morgan Kaufmann Publishers Inc. 